\title{
OPEN Two meta-analyses of the association between atopic diseases and core symptoms of attention deficit hyperactivity disorder
}

\author{
Yu-Chieh Chuang ${ }^{1}$, Ching-Yun Wang ${ }^{2}$, Wei-Lieh Huang ${ }^{3,4,5,6}$, Liang-Jen Wang ${ }^{7,8}$, \\ Ho-Chang Kuo ${ }^{9,10}$, Yang-Ching Chen ${ }^{11,12}$ \& Yu-Jui Huang ${ }^{13 凶}$
}

Studies in the field of neuroscience and psychology have hypothesized that a causal association exists between atopic diseases and attention-deficit/hyperactivity disorder (ADHD). Previous systematic reviews and meta-analyses have reported a higher risk of ADHD in children with atopic diseases; however, the relationship between ADHD symptoms and atopic diseases remains unclear. We systematically reviewed observational cross-sectional and longitudinal studies to investigate the relationship between atopic diseases and ADHD symptom severity (hyperactivity/impulsivity and inattention). The majority of studies showed a statistically significant association between atopic diseases and both ADHD symptoms, with substantial heterogeneity in the outcome of hyperactivity/ impulsivity. Remarkably decreased heterogeneity and statistical significance were observed in the second meta-analysis of ADHD-related behavior symptoms in atopic patients without ADHD. Our study indicated that atopic diseases not only associated with ADHD but also ADHD symptoms severity. This association was even observed in children with subthreshold ADHD, indicating that atopic diseases may play a role in the spectrum of ADHD symptom severity. Trial registration: This study was registered on PROSPERO (registration ID: CRD42020213219).

In the past decade, atopic diseases have been a cause of increased concern because of their substantially high prevalence (approximately 20 to $40 \%$ ) worldwide ${ }^{1}$, with more prominent increasing trend in developing countries ${ }^{2}$. Atopic march, defined as the development of atopic dermatitis in infancy and subsequent allergic rhinitis and asthma in later childhood, can cause fatigue; attention, learning, and memory deficits; and depression. Moreover, atopic diseases have been reported to considerably affect children's sleep, school performance, development, and quality of life ${ }^{3,4}$. In addition, recent studies have reported that the parents or main caregivers of children with atopic diseases, particularly those with multiple atopic diseases, experience considerable stress and psychosocial burden $^{4-8}$ Thus, increasing public attention has been focused on the management of atopic diseases ${ }^{8}$.

Attention-deficit/hyperactivity disorder (ADHD) is among the most common neuropsychiatric disorders in children and adolescents, with a prevalence of approximately $5 \%$. ADHD is characterized by hyperactivity,

\footnotetext{
${ }^{1}$ Department of General Medicine, Shuang Ho Hospital, Taipei Medical University, New Taipei City, Taiwan. ${ }^{2}$ Department of Pediatrics, Taipei Medical University Hospital, Taipei, Taiwan. ${ }^{3}$ Department of Psychiatry, National Taiwan University Hospital Yunlin Branch, Yunlin, Taiwan. ${ }^{4}$ Department of Psychiatry, National Taiwan University Hospital, Taipei, Taiwan. ${ }^{5}$ Department of Psychiatry, College of Medicine, National Taiwan University, Taipei, Taiwan. ${ }^{6}$ Graduate Institute of Clinical Medicine, College of Medicine, National Taiwan University, Taipei, Taiwan. 'Department of Child and Adolescent Psychiatry, Kaohsiung Chang Gung Memorial Hospital and Chang Gung University College of Medicine, Kaohsiung, Taiwan. ${ }^{8}$ School of Medicine, College of Medicine, Chang Gung University, Taoyuan, Taiwan. ${ }^{9}$ Department of Pediatrics, Kaohsiung Chang Gung Memorial Hospital and Chang Gung University College of Medicine, Kaohsiung, Taiwan. ${ }^{10}$ Kawasaki Disease Center, Kaohsiung Chang Gung Memorial Hospital, Kaohsiung, Taiwan. ${ }^{11}$ Department of Family Medicine, Taipei Medical University Hospital, Taipei, Taiwan. ${ }^{12}$ Department of Family Medicine, School of Medicine, College of Medicine, Taipei Medical University, Taipei, Taiwan. ${ }^{13}$ Department of Psychiatry and Psychiatric Research Center, Taipei Medical University Hospital, Taipei, Taiwan. ${ }^{\circledR}$ email: yujui0515@gmail.com
} 
impulsivity, and inattention that lead to not only functional impairment but also executive function impairment and emotional dysregulation ${ }^{9,10}$. ADHD can be caused by multiple factors (e.g., genetic, epigenetic, and environmental). Moreover, studies have indicated that ADHD occurs due to immature brain development, and it may also be accompanied by other neurobehavioral developmental delays and even intellectual disability ${ }^{10,11}$. Many studies have reported that ADHD symptoms were still observed in adulthood in a majority of patients with ADHD, even in those with adult ADHD who did not meet the criteria for ADHD in their childhood ${ }^{12-14}$. Adult patients with ADHD experience a financial burden and may even require considerable support ${ }^{14}$. These findings indicate that ADHD is a matter of considerable concern in child and adolescent psychiatry.

A previous study investigated the relationship between atopic diseases and $\mathrm{ADHD}^{15}$. A higher risk of $\mathrm{ADHD}$ was noted in patients with all three atopic diseases, namely atopic dermatitis, allergic rhinitis, and asthma (i.e., atopic march $)^{16,17}$. In addition, children with both atopic diseases and ADHD had an increased risk of developing more severe ADHD symptoms compared with those with only $\mathrm{ADHD}^{18}$. Also, the association between atopic diseases and ADHD-related behavioral symptoms ${ }^{19}$, which some researches mentioned as subthreshold ADHD ${ }^{20}$, has been investigated recently. Although several meta-analyses and systematic reviews have strongly supported the relationship between atopic diseases and $\mathrm{ADHD}^{17,21,22}$, they still had many limitations. The heterogeneity observed in some systematic reviews might have affected their findings; for instance, some reviews did not include studies with standard inclusion and exclusion criteria, and whether the atopic and unexposed groups included patients diagnosed as having ADHD remained unclear ${ }^{16,21}$. In addition, few studies have reported a relationship between atopic diseases and subthreshold ADHD. Moreover, no systematic review and meta-analysis has examined whether atopic diseases affect the severity of both hyperactivity/impulsivity and inattention-the two main symptoms and diagnostic criteria related to ADHD.

In this systematic review and meta-analysis, we reviewed studies examining the relationship between atopic diseases and the severity of ADHD symptoms including hyperactivity/impulsivity and inattention. In addition, we explored more precise value of this relationship by defining inclusion and exclusion criteria for groups. By doing so, we could exclude some confounding effects and provide additional information regarding the relationship between atopic diseases and subthreshold ADHD; this involved examining the data of children without a previous diagnosis of ADHD but who had an association of developing more severe ADHD symptoms.

\section{Results}

Search results. Through our literature search, we identified 4722 studies $(2872,1504,345$, and 1 from PubMed + Medline, Embase, Psycinfo, and register, respectively). Fifteen additional studies were identified through other sources. Using EndNote, we found that 671 studies were duplicates and we thus excluded them. Subsequently, we screened the titles and abstract for eligibility of the remaining 4066 studies. During title and abstract screening, we removed 630 because they did not include a relevant unexposed group and were letters or replies. Additionally, we excluded 1077 studies because they were not original research. Moreover, 1222 studies were excluded because they were not relevant to our research. Subsequently, after reviewing the full texts of the remaining 1136 studies, we excluded 1098 studies, 78 of which had missing data or full texts, 811 had nonrelevance, and 210 were not case-control, cross-sectional, or cohort studies. Finally, we included the remaining 38 studies in this systematic review for qualitative synthesis, and 16 of them were included in the quantitative review and meta-analysis. The procedure for study inclusion is shown in Appendix B in the Supplementary Materials.

Qualitative systematic review. In 38 studies including 117,181,049 participants in our qualitative systemic review, the researchers examined the relationship between atopic diseases and ADHD symptoms severity by displaying prevalence of ADHD in patients with atopy, odds ratios (OR) of Attention deficit disorder (ADD)/ ADHD in children with atopy, or calculating scores for hyperactivity/impulsivity, inattention, or total ADHD symptoms. Table 1 lists the characteristics of included studies. Most of the studies assessed atopic diseases by using parental questionnaires or based on their diagnosis by physicians or dermatologists according to specific diagnostic criteria, including the Global Initiative for Asthma guidelines, Allergic Rhinitis and its Impact on Asthma guidelines, and UK Working Party criteria. Seven study groups conducted an advanced additional test for atopy such as the skin-prick test and blood sampling for serum-specific IgE (MAST or Phadiatop tests). The included studies used different outcome scales including the Conners' Parent Rating Scale; ADHD Rating Scale; Swanson, Nolan, and Pelham IV Scale; Child Behavior Checklist, Early Childhood Inventory-4, the German ADHD Rating scale (FBB-HKS), and the Strengths and Difficulties Questionnaire (SDQ). Despite the variability in outcome scales, most scales were designed on the basis of the DSM-IV criteria. Eight studies adopted the SDQ, which is not derived from the DSM-IV criteria; however, the SDQ has been reported to be relevant to the DSM-IV ${ }^{23-25}$. Most of the studies investigated the effect of confounding factors, including age, sex, multiple atopic diseases, the comorbidity with ADHD, severity of atopic diseases, persistent atopic diseases, and stressful parenting. Nineteen of the twenty studies examining the outcome of total ADHD symptoms reported a significant relationship between the severity of ADHD symptoms and atopic diseases. Furthermore, 9 of the 15 studies examining the outcome of hyperactivity and 13 of the 16 studies examining the outcome of inattention demonstrated a significant relationship between the severity of the examined ADHD symptoms and atopic diseases.

Three of the four studies reported a significant association between the comorbidity of ADHD and atopic diseases in patients with ADHD. Ten studies reported a significantly positive correlation among atopic diseases, sleep disturbance, and ADHD or an increased risk of ADHD in children with atopic diseases. One research group ${ }^{26}$ performed structural equation modeling to determine interactions among eczema, asthma, rhinitis, sleep, and behavior disorders. In three studies, the computerized comprehensive attention test (CAT) was performed, a more objective and precise measurement tool, to assess attention deficit, and they obtained results similar to those of other studies, thus supporting our hypothesis that atopy increased ADHD symptoms severity. In addition, 


\begin{tabular}{|c|c|c|c|c|c|c|c|c|c|}
\hline Study name & $\begin{array}{l}\text { Outcome (95\% } \\
\text { CI) }\end{array}$ & $\begin{array}{l}\text { Study } \\
\text { design }\end{array}$ & $\begin{array}{l}\text { Country/ } \\
\text { City }\end{array}$ & Ethnicity & $\begin{array}{l}\text { Number of participants/ } \\
\text { Exposed group/Unexposed } \\
\text { group }\end{array}$ & $\begin{array}{l}\text { Age (mean of the } \\
\text { exposed group)/sex } \\
\text { (male, \%) }\end{array}$ & $\begin{array}{l}\text { Diagnostic } \\
\text { criteria for } \\
\text { ADHD }\end{array}$ & \begin{tabular}{|l|} 
Atopic \\
disease/ \\
method \\
used for \\
assessing \\
atopic \\
diseases \\
\end{tabular} & $\begin{array}{l}\text { Adjustment } \\
\text { for } \\
\text { confounding } \\
\text { factors }\end{array}$ \\
\hline $\begin{array}{l}\text { Yuksel et al. } \\
2008^{58}\end{array}$ & $\begin{array}{l}\text { Continuous data } \\
\text { for } \mathrm{HI} \text { and } \mathrm{IN}^{\star}\end{array}$ & $\begin{array}{l}\text { Cross- } \\
\text { sectional }\end{array}$ & $\begin{array}{l}\text { Manisa, } \\
\text { Turkey }\end{array}$ & $\begin{array}{l}\text { Not } \\
\text { reported }\end{array}$ & $100 / 62 / 38$ & $7-12$ years $(9.2) / 57 \%$ & DSM-IV & $\begin{array}{l}\text { Asthma/ } \\
\text { Physician's } \\
\text { diagnosis } \\
\text { (GINA } \\
\text { 2006) }\end{array}$ & No \\
\hline $\begin{array}{l}\text { Camfferman } \\
\text { et al. } 2010^{26}\end{array}$ & $\begin{array}{l}\text { Continuous data } \\
\text { for } \mathrm{HI}^{*} \text { and total } \\
\mathrm{ADHD} \text { symp- } \\
\text { toms }\end{array}$ & $\begin{array}{l}\text { Cross- } \\
\text { sectional }\end{array}$ & $\begin{array}{l}\text { South } \\
\text { Australia, } \\
\text { Australia }\end{array}$ & $\begin{array}{l}\text { Not } \\
\text { reported }\end{array}$ & $107 / 77 / 30$ & $\begin{array}{l}6-16 \text { years } \\
(9.9) / 46.72 \%\end{array}$ & $\begin{array}{l}\text { Conner's } \\
\text { Parent Rat- } \\
\text { ing Scale- } \\
\text { Revised }\end{array}$ & $\begin{array}{l}\text { Atopic } \\
\text { dermatitis/ } \\
\text { Physician's } \\
\text { diagnosis } \\
\text { (Hanifin } \\
\text { and Raika } \\
\text { criteria) }\end{array}$ & Yes \\
\hline $\begin{array}{l}\text { Chang et al. } \\
2013^{59}\end{array}$ & $\begin{array}{l}\text { Continuous data } \\
\text { for HI and IN }\end{array}$ & $\begin{array}{l}\text { Cross- } \\
\text { sectional }\end{array}$ & $\begin{array}{l}\text { Seoul and } \\
\text { Ilsan and } \\
\text { Gwacheon } \\
\text { in } \\
\text { Gyeonggi- } \\
\text { do province } \\
\text { in Korea }\end{array}$ & $\begin{array}{l}\text { Not } \\
\text { reported }\end{array}$ & 575 & $3-7$ years $(4.8) / 51.3 \%$ & CBCL & \begin{tabular}{|l|} 
Atopic \\
dermatitis, \\
allergic \\
rhinitis, and \\
asthma/ \\
Physician's \\
diagnosis \\
and ques- \\
tionnaire \\
(ISAAC \\
question- \\
naire and \\
SCORAD \\
index) \\
\end{tabular} & No \\
\hline $\begin{array}{l}\text { Goodwin } \\
\text { et al. } 2013^{60}\end{array}$ & $\begin{array}{l}\text { OR of ADHD } \\
\text { in children with } \\
\text { asthma }{ }^{*}(\text { different } \\
\text { grades of severity } \\
{[\text { moderate }} \\
\text { sistent }[\text { remission- } \\
\left.\left.\text { and late onset }{ }^{*}\right]\right)\end{array}$ & Cohort & $\begin{array}{l}\text { Perth, } \\
\text { Western } \\
\text { Australia }\end{array}$ & $\begin{array}{l}\text { Not } \\
\text { reported }\end{array}$ & $2193 / 390 / 1803$ & $\begin{array}{l}5,8,10,14, \text { and } \\
17 \text { years } 62.56 \%\end{array}$ & CBCL & $\begin{array}{l}\text { Asthma/ } \\
\text { Physician's } \\
\text { diagnosis } \\
\text { (Australian } \\
\text { Asthma } \\
\text { Handbook) }\end{array}$ & Yes \\
\hline $\begin{array}{l}\text { Kim et al. } \\
2014^{61}\end{array}$ & $\begin{array}{l}\text { Continuous data } \\
\text { for impulsivity } \\
\text { and inattention } \\
\text { (divided attention } \\
\text { task }^{*} \text { ) }\end{array}$ & Cohort & Korea & $\begin{array}{l}\text { Not } \\
\text { reported }\end{array}$ & $1036 / 797 / 239$ & $\begin{array}{l}\text { 3-16 years } \\
(11.4) / 69.88 \%\end{array}$ & $\begin{array}{l}\text { Computer- } \\
\text { ized com- } \\
\text { prehensive } \\
\text { attention } \\
\text { test }\end{array}$ & $\begin{array}{l}\text { Allergic } \\
\text { rhinitis/skin } \\
\text { prick test }\end{array}$ & Yes \\
\hline $\begin{array}{l}\text { Lee et al. } \\
2014^{62}\end{array}$ & $\begin{array}{l}\text { Continuous data } \\
\text { for } \mathrm{HI} \text { and } \mathrm{IN}^{\star}\end{array}$ & $\begin{array}{l}\text { Cross- } \\
\text { sectional }\end{array}$ & Seoul, Korea & $\begin{array}{l}\text { Not } \\
\text { reported }\end{array}$ & $160 / 87 / 73$ & $\begin{array}{l}6-13 \text { years } \\
(8.49) / 39.4 \%\end{array}$ & DSM-IV & $\begin{array}{l}\text { Allergic } \\
\text { rhinitis/ } \\
\text { physician's } \\
\text { diagnosis } \\
\text { (ARIA } \\
\text { guideline) } \\
\end{array}$ & Yes \\
\hline $\begin{array}{l}\text { Yang et al. } \\
2014^{63}\end{array}$ & $\begin{array}{l}\text { Continuous data } \\
\text { for } \mathrm{HI}^{\star} \text { and } \mathrm{IN}^{\star}\end{array}$ & $\begin{array}{l}\text { Cross- } \\
\text { sectional }\end{array}$ & $\begin{array}{l}\text { Taipei, } \\
\text { Taiwan }\end{array}$ & $\begin{array}{l}\text { Not } \\
\text { reported }\end{array}$ & $122 / 93 / 29$ & $\begin{array}{l}\text { 6-14 years } \\
(10.78) / 52.2 \%\end{array}$ & $\begin{array}{l}\text { DSM-IV- } \\
\text { TR }\end{array}$ & \begin{tabular}{|l|} 
Allergic \\
rhinitis/ \\
Physician's \\
diagnosis \\
(ARIA \\
2008) + IgE \\
test (MAST \\
or Phadi- \\
atop tests)
\end{tabular} & Yes \\
\hline $\begin{array}{l}\text { Catal et al. } \\
2016^{64}\end{array}$ & $\begin{array}{l}\text { Continuous data } \\
\text { for total ADHD } \\
\text { symptoms }{ }^{\star} \text { and } \\
\text { percentage of psy- } \\
\text { chiatric disorders } \\
\text { determined by } \\
\text { ECI- } 4^{*}\end{array}$ & $\begin{array}{l}\text { Cross- } \\
\text { sectional }\end{array}$ & $\begin{array}{l}\text { Malatya, } \\
\text { Samsun, } \\
\text { Aydın, } \\
\text { Istanbul, } \\
\text { Turkey }\end{array}$ & $\begin{array}{l}\text { Not } \\
\text { reported }\end{array}$ & $154 / 80 / 74$ & $3-5$ years $(4.03) / 51.3 \%$ & ECI-4 & \begin{tabular}{|l|} 
Atopic \\
dermatitis/ \\
Physician's \\
diagnosis \\
(Hanifin \\
and Rajka \\
criteria) \\
\end{tabular} & No \\
\hline $\begin{array}{l}\text { Hammer- } \\
\text { Helmich } \\
\text { et al. } 2016^{65}\end{array}$ & $\begin{array}{l}\text { Continuous data } \\
\text { for total ADHD } \\
\text { symptoms }{ }^{\star} \odot\end{array}$ & $\begin{array}{l}\text { Cross- } \\
\text { sectional }\end{array}$ & $\begin{array}{l}\text { Copen- } \\
\text { hagen, } \\
\text { Denmark }\end{array}$ & $\begin{array}{l}\text { Not } \\
\text { reported }\end{array}$ & $9036 / 2433 / 6603$ & $3-15$ years $(\mathrm{NA}) / 49.5 \%$ & SDQ & $\begin{array}{l}\text { Eczema, } \\
\text { asthma, and } \\
\text { hay fever/ } \\
\text { Question- } \\
\text { naire (UK } \\
\text { Working } \\
\text { Party } \\
\text { Criteria) }\end{array}$ & Yes \\
\hline $\begin{array}{l}\text { Strom et al. } \\
2016^{66}\end{array}$ & $\begin{array}{l}\text { OR of ADD/ } \\
\text { ADHD in chil- } \\
\text { dren with atopic } \\
\text { dermatitis }^{*}\end{array}$ & $\begin{array}{l}\text { Cross- } \\
\text { sectional }\end{array}$ & $\begin{array}{l}\text { U.S. Bureau } \\
\text { of Census }\end{array}$ & $\begin{array}{l}\text { Not } \\
\text { reported }\end{array}$ & $180,799 / 17,277 / 163,522$ & $\begin{array}{l}<18 \text { years } \\
\text { (NA) } / 49.94 \%\end{array}$ & $\begin{array}{l}\text { Question- } \\
\text { naire }\end{array}$ & $\begin{array}{l}\text { Atopic } \\
\text { dermatitis, } \\
\text { asthma, } \\
\text { and allergic } \\
\text { rhinitis/ } \\
\text { question- } \\
\text { naire }\end{array}$ & Yes \\
\hline \multicolumn{10}{|l|}{ Continued } \\
\hline
\end{tabular}




\begin{tabular}{|c|c|c|c|c|c|c|c|c|c|}
\hline Study name & $\begin{array}{l}\text { Outcome (95\% } \\
\text { CI) }\end{array}$ & $\begin{array}{l}\text { Study } \\
\text { design }\end{array}$ & $\begin{array}{l}\text { Country/ } \\
\text { City }\end{array}$ & Ethnicity & $\begin{array}{l}\text { Number of participants/ } \\
\text { Exposed group/Unexposed } \\
\text { group }\end{array}$ & $\begin{array}{l}\text { Age (mean of the } \\
\text { exposed group)/sex } \\
\text { (male, \%) }\end{array}$ & $\begin{array}{l}\text { Diagnostic } \\
\text { criteria for } \\
\text { ADHD }\end{array}$ & \begin{tabular}{|l|} 
Atopic \\
disease/ \\
method \\
used for \\
assessing \\
atopic \\
diseases \\
\end{tabular} & $\begin{array}{l}\text { Adjustment } \\
\text { for } \\
\text { confounding } \\
\text { factors }\end{array}$ \\
\hline $\begin{array}{l}\text { Yang et al. } \\
2016^{67}\end{array}$ & $\begin{array}{l}\text { Continuous data } \\
\text { for } \mathrm{HI}^{\star} \text { and } \mathrm{IN}^{\star}\end{array}$ & $\begin{array}{l}\text { Case-con- } \\
\text { trol }\end{array}$ & $\begin{array}{l}\text { Taipei, } \\
\text { Taiwan }\end{array}$ & $\begin{array}{l}\text { Not } \\
\text { reported }\end{array}$ & $99 / 68 / 31$ & $\begin{array}{l}6-14 \text { years } \\
(9.25) / 82.4 \%\end{array}$ & DSM-IV & $\begin{array}{l}\text { Allergic } \\
\text { rhinitis/ } \\
\text { physician's } \\
\text { diagno- } \\
\text { sis + IgE test } \\
\text { (MAST or } \\
\text { Phadiatop } \\
\text { tests) }\end{array}$ & Yes \\
\hline $\begin{array}{l}\text { Feng et al. } \\
2017^{68}\end{array}$ & $\begin{array}{l}\text { Continuous data } \\
\text { for } \mathrm{HI}^{\star} \text { and } \mathrm{IN}^{\star}\end{array}$ & $\begin{array}{l}\text { Cross- } \\
\text { sectional }\end{array}$ & $\begin{array}{l}\text { Wenzhou, } \\
\text { China }\end{array}$ & $\begin{array}{l}\text { Not } \\
\text { reported }\end{array}$ & $643 / 248 / 320$ & $\begin{array}{l}6-12 \text { years } \\
(9.25) / 51.9 \%\end{array}$ & DSM-IV & \begin{tabular}{|l|} 
Allergic \\
rhinitis/ \\
physician's \\
diagnosis \\
(ARIA \\
2008)+skin \\
prick test
\end{tabular} & Yes \\
\hline $\begin{array}{l}\text { Lin et al. } \\
2017^{45}\end{array}$ & \begin{tabular}{|l|} 
OR of ADHD \\
(inattentive/ \\
Hyperactive \\
impulsive/com- \\
bined) in children \\
with atopic der- \\
matitis, asthma, \\
allergic rhinitis \\
(fever/active) $^{*}$
\end{tabular} & $\begin{array}{l}\text { Cross- } \\
\text { sectional }\end{array}$ & Taiwan & $\begin{array}{l}\text { Han Chi- } \\
\text { nese }\end{array}$ & $2896 / 2235 / 661$ & $9-10$ years $(10) / 50.5 \%$ & SNAP-IV & $\begin{array}{l}\text { Atopic } \\
\text { dermatitis, } \\
\text { asthma, and } \\
\text { allergic rhi- } \\
\text { nitis/ques- } \\
\text { tionnaire } \\
\text { (ISAAC } \\
\text { question- } \\
\text { naire) }\end{array}$ & Yes \\
\hline $\begin{array}{l}\text { Abd El- } \\
\text { Hamid et al. } \\
2018^{69}\end{array}$ & $\begin{array}{l}\text { Prevalence of } \\
\text { different grades of } \\
\text { ADHD symptoms } \\
\text { (mild, moderate, } \\
\text { and severe) in } \\
\text { children with or } \\
\text { without atopic } \\
\text { diseases }^{*}\end{array}$ & $\begin{array}{l}\text { Case-con- } \\
\text { trol }\end{array}$ & Cairo, Egypt & $\begin{array}{l}\text { Not } \\
\text { reported }\end{array}$ & $160 / 100 / 60$ & $\begin{array}{l}6-12 \text { years } \\
(8.54) / 81.25 \%\end{array}$ & DSM-IV & \begin{tabular}{|l|} 
Allergic \\
diseases/ \\
physician's \\
diagnosis, \\
skin prick \\
test, and \\
total IgE \\
enzyme \\
immunoas- \\
say (ELISA)
\end{tabular} & No \\
\hline $\begin{array}{l}\text { Kuniyoshi } \\
\text { et al. } 2018^{70}\end{array}$ & $\begin{array}{l}\text { Continuous data } \\
\text { for total ADHD } \\
\text { symptoms } \bigodot^{*}\end{array}$ & $\begin{array}{l}\text { Cross- } \\
\text { sectional }\end{array}$ & $\begin{array}{l}\text { Miyagi } \\
\text { Prefecture, } \\
\text { Japan }\end{array}$ & $\begin{array}{l}\text { Not } \\
\text { reported }\end{array}$ & $9954 / 1641 / 8313$ & $\begin{array}{l}7-14 \text { years } \\
(10.42) / 49.9 \%\end{array}$ & SDQ & $\begin{array}{l}\text { Eczema/ } \\
\text { ISAAC } \\
\text { (Eczema } \\
\text { symptom } \\
\text { question- } \\
\text { naire) } \\
\end{array}$ & Yes \\
\hline $\begin{array}{l}\text { Schmitt } \\
\text { et al. } 2018^{6}\end{array}$ & $\begin{array}{l}\text { Continuous data } \\
\text { for HI and } \mathrm{IN}^{*}\end{array}$ & $\begin{array}{l}\text { Case-con- } \\
\text { trol }\end{array}$ & $\begin{array}{l}\text { Dresden, } \\
\text { Germany }\end{array}$ & $\begin{array}{l}\text { Not } \\
\text { reported }\end{array}$ & $89 / 35 / 45$ & $6-12$ years $(9.9) / 62.9 \%$ & ICD-10 & $\begin{array}{l}\text { Atopic der- } \\
\text { matitis/der- } \\
\text { matologist's } \\
\text { diagnosis } \\
\text { (UK Work- } \\
\text { ing Party } \\
\text { Criteria) }\end{array}$ & Yes \\
\hline $\begin{array}{l}\text { Cices et al. } \\
2019^{71}\end{array}$ & $\begin{array}{l}\text { Continuous data } \\
\text { for HI and } \mathrm{IN}^{*}\end{array}$ & $\begin{array}{l}\text { Case-con- } \\
\text { trol }\end{array}$ & $\begin{array}{l}\text { US, } \\
\text { Chicago, } \\
\text { Illinois }\end{array}$ & \begin{tabular}{|l|} 
Caucasian \\
$(31.43 \%)$ \\
African \\
American \\
$(22.86 \%)$ \\
Hispanic \\
$(20 \%)$ Asian \\
$(20 \%)$ Other \\
(5.71\%) \\
\end{tabular} & $35 / 17 / 18$ & $\begin{array}{l}\text { 6-16 years } \\
(11.07) / 65.7 \%\end{array}$ & $\begin{array}{l}\text { Stand- } \\
\text { ardized } \\
\text { Vanderbilt } \\
\text { question- } \\
\text { naires }\end{array}$ & $\begin{array}{l}\text { Atopic } \\
\text { dermatitis/ } \\
\text { SCORAD }\end{array}$ & Yes \\
\hline $\begin{array}{l}\text { Tajdini et al. } \\
2019^{72}\end{array}$ & $\begin{array}{l}\text { Prevalence of } \\
\text { ADHD in patients } \\
\text { with asthma and } \\
\text { controls* }\end{array}$ & $\begin{array}{l}\text { Case-con- } \\
\text { trol }\end{array}$ & Tehran, Iran & $\begin{array}{l}\text { Not } \\
\text { reported }\end{array}$ & $171 / 79 / 92$ & $5-11$ years $(7.54) / 57 \%$ & \begin{tabular}{|l|} 
Child \\
Symptom \\
Inventory-4 \\
(CSI-4) \\
\end{tabular} & $\begin{array}{l}\text { Asthma/ } \\
\text { respiratory } \\
\text { function } \\
\text { test }\end{array}$ & Yes \\
\hline $\begin{array}{l}\text { Zhou et al. } \\
2019^{73}\end{array}$ & $\begin{array}{l}\text { Prevalence of } \\
\text { ADHD in patients } \\
\text { with asthma and } \\
\text { controls }^{*}\end{array}$ & $\begin{array}{l}\text { Cross- } \\
\text { sectional }\end{array}$ & $\begin{array}{l}\text { Guiyang, } \\
\text { China }\end{array}$ & $\begin{array}{l}\text { Han } \\
\text { (77.59\%) }\end{array}$ & $522 / 261 / 261$ & \begin{tabular}{|l} 
6-16 years \\
$(9.35) / 61.69 \%$
\end{tabular} & $\begin{array}{l}\text { Mini-Inter- } \\
\text { national } \\
\text { Neuropsy- } \\
\text { chiatric } \\
\text { Interview } \\
\text { for children } \\
\text { and adoles- } \\
\text { cents }\end{array}$ & \begin{tabular}{|l|} 
Asthma/ \\
physician's \\
diagnosis \\
(Bronchial \\
Asthma \\
Diagnostic \\
and Preven- \\
tion Guide \\
for Children \\
{$\left[\begin{array}{l}\text { [2016 ver- } \\
\text { sion]) }\end{array}\right.$} \\
\end{tabular} & Yes \\
\hline
\end{tabular}




\begin{tabular}{|c|c|c|c|c|c|c|c|c|c|}
\hline Study name & $\begin{array}{l}\text { Outcome (95\% } \\
\text { CI) }\end{array}$ & $\begin{array}{l}\text { Study } \\
\text { design }\end{array}$ & $\begin{array}{l}\text { Country/ } \\
\text { City }\end{array}$ & Ethnicity & $\begin{array}{l}\text { Number of participants/ } \\
\text { Exposed group/Unexposed } \\
\text { group }\end{array}$ & $\begin{array}{l}\text { Age (mean of the } \\
\text { exposed group)/sex } \\
\text { (male, \%) }\end{array}$ & $\begin{array}{l}\text { Diagnostic } \\
\text { criteria for } \\
\text { ADHD }\end{array}$ & \begin{tabular}{|l|} 
Atopic \\
disease/ \\
method \\
used for \\
assessing \\
atopic \\
diseases
\end{tabular} & $\begin{array}{l}\text { Adjustment } \\
\text { for } \\
\text { confounding } \\
\text { factors }\end{array}$ \\
\hline $\begin{array}{l}\text { Feng et al. } \\
2020^{74}\end{array}$ & $\begin{array}{l}\text { Continuous data } \\
\text { for } \mathrm{HI}^{*} \text { and } \mathrm{IN}^{*}\end{array}$ & $\begin{array}{l}\text { Cross- } \\
\text { sectional }\end{array}$ & $\begin{array}{l}\text { Chongqing, } \\
\text { China }\end{array}$ & $\begin{array}{l}\text { Not } \\
\text { reported }\end{array}$ & $273 / 89 / 184$ & $\begin{array}{l}6-12 \text { years } \\
(8.3) / 62.63 \%\end{array}$ & SNAP-IV & $\begin{array}{l}\text { Atopic } \\
\text { dermatitis/ } \\
\text { dermatolo- } \\
\text { gist's diag- } \\
\text { nosis (UK } \\
\text { criteria) }\end{array}$ & Yes \\
\hline $\begin{array}{l}\text { Fuhrmann } \\
\text { et al. } 2020^{75}\end{array}$ & $\begin{array}{l}\text { HR of ADD/ } \\
\text { ADHD in chil- } \\
\text { dren with atopic } \\
\text { dermatitis }^{*}\end{array}$ & Cohort & Germany & $\begin{array}{l}\text { Not } \\
\text { reported }\end{array}$ & $37,235 / 9257 / 27,978$ & $\begin{array}{l}\text { From } 0 \text { to } \\
7-9 \text { years } / 51.7 \%\end{array}$ & ICD-10 & $\begin{array}{l}\text { Atopic } \\
\text { dermatitis/ } \\
\text { ICD-10 }\end{array}$ & Yes \\
\hline $\begin{array}{l}\text { Guo et al. } \\
2020^{76}\end{array}$ & $\begin{array}{l}\text { Continuous data } \\
\text { for } \mathrm{HI}(\mathrm{AR}, \mathrm{AD} \text {, } \\
\text { and asthma) and } \\
\mathrm{IN}\left(\mathrm{AR}^{\star}, \mathrm{AD} \text {, and }\right. \\
\text { asthma } \mathrm{a}^{*}\end{array}$ & Cohort & $\begin{array}{l}\text { Kaohsiung, } \\
\text { Taiwan }\end{array}$ & $\begin{array}{l}\text { Not } \\
\text { reported }\end{array}$ & 97 & 6 years $/ 54.6 \%$ & SNAP-IV & $\begin{array}{l}\text { Atopic } \\
\text { dermatitis, } \\
\text { asthma, and } \\
\text { allergic rhi- } \\
\text { nitis/ISAAC } \\
\text { question- } \\
\text { naire, } \\
\text { physician's } \\
\text { diagnosis } \\
\text { (Hani- } \\
\text { fin-Rajka } \\
\text { criteria), } \\
\text { and IgE test }\end{array}$ & Yes \\
\hline $\begin{array}{l}\text { Huang et al. } \\
2020^{77}\end{array}$ & $\begin{array}{l}\text { OR of ADD/ } \\
\text { ADHD in chil- } \\
\text { dren with atopic } \\
\text { dermatitis }^{*}\end{array}$ & $\begin{array}{l}\text { Cross- } \\
\text { sectional }\end{array}$ & $\begin{array}{l}\text { US } \\
\text { Northeast } \\
(17.96 \%) \\
\text { North } \\
\text { Central } \\
(19.33 \%) \\
\text { South } \\
(45.83 \%) \\
\text { West } \\
(16.42 \%) \\
\text { Unknown } \\
(0.47 \%)\end{array}$ & $\begin{array}{l}\text { Not } \\
\text { reported }\end{array}$ & $203,533 / 86,969 / 116,564$ & $\begin{array}{l}0-17 \text { years } \\
(5.3) / 51.74 \%\end{array}$ & $\begin{array}{l}\text { ICD- } \\
10-C M\end{array}$ & $\begin{array}{l}\text { Atopic der- } \\
\text { matitis/IBM } \\
\text { MarketScan } \\
\text { Commercial } \\
\text { Claims } \\
\text { database } \\
\text { (ICD- } \\
\text { 10-CM code } \\
\text { for AD: } \\
\text { L20*) }\end{array}$ & Yes \\
\hline $\begin{array}{l}\text { Jackson- } \\
\text { Cowan et al. } \\
2020^{78}\end{array}$ & $\begin{array}{l}\text { OR of ADD/ } \\
\text { ADHD in chil- } \\
\text { dren with atopic } \\
\text { dermatitis }^{*}\end{array}$ & $\begin{array}{l}\text { Cross- } \\
\text { sectional }\end{array}$ & $\begin{array}{l}\text { US } \\
\text { Northeast } \\
\text { (15.73\%) } \\
\text { North } \\
\text { Central } \\
(20.27 \%) \\
\text { South } \\
(36.21 \%) \\
\text { West } \\
(27.78 \%)\end{array}$ & $\begin{array}{l}\text { White } \\
(48.44 \%) \\
\text { Black } \\
(15.38 \%) \\
\text { Hispanic } \\
(27.86 \%) \\
\text { Asian } \\
(6.06 \%) \\
\text { Native } \\
\text { American } \\
(0.97 \%) \\
\text { Other } \\
(1.29 \%) \\
\end{array}$ & $109,482 / 13,398 / 96,084$ & $2-17$ years $/ 51.48 \%$ & $\begin{array}{l}\text { Question- } \\
\text { naire }\end{array}$ & $\begin{array}{l}\text { Atopic } \\
\text { dermatitis/ } \\
\text { question- } \\
\text { naire }\end{array}$ & Yes \\
\hline $\begin{array}{l}\text { Kuo et al. } \\
2020^{41}\end{array}$ & $\begin{array}{l}\text { Continuous data } \\
\text { for } \mathrm{HI}^{*} \text { and IN }\end{array}$ & $\begin{array}{l}\text { Cross- } \\
\text { sectional }\end{array}$ & $\begin{array}{l}\text { Kaohsiung, } \\
\text { Taiwan }\end{array}$ & $\begin{array}{l}\text { Not } \\
\text { reported }\end{array}$ & $191 / 109 / 92$ & $(6.59) / 54.44 \%$ & SNAP-IV & $\begin{array}{l}\text { Atopic } \\
\text { dermatitis, } \\
\text { asthma, } \\
\text { and allergic } \\
\text { rhinitis/ } \\
\text { physician's } \\
\text { diagnosis }\end{array}$ & Yes \\
\hline $\begin{array}{l}\text { Minatoya } \\
\text { et al. } 2020^{79}\end{array}$ & $\begin{array}{l}\text { Continuous data } \\
\text { for total ADHD } \\
\text { symptoms* } \\
\end{array}$ & $\begin{array}{l}\text { Cross- } \\
\text { sectional }\end{array}$ & $\begin{array}{l}\text { Hokkaido, } \\
\text { Japan }\end{array}$ & $\begin{array}{l}\text { Not } \\
\text { reported }\end{array}$ & $3862 / 799 / 3063$ & $(5.28) / 49.9 \%$ & SDQ & $\begin{array}{l}\text { Atopic } \\
\text { dermatitis/ } \\
\text { ISAAC }\end{array}$ & Yes \\
\hline $\begin{array}{l}\text { Montalbano } \\
\text { et al. } 2020^{80}\end{array}$ & $\begin{array}{l}\text { Continuous data } \\
\text { for total ADHD } \\
\text { symptoms* }\end{array}$ & $\begin{array}{l}\text { Cross- } \\
\text { sectional }\end{array}$ & $\begin{array}{l}\text { Florence, } \\
\text { Italy }\end{array}$ & $\begin{array}{l}\text { Not } \\
\text { reported }\end{array}$ & 141 & $6-11$ years $(10) / 61.7 \%$ & SDQ & $\begin{array}{l}\text { Asthma/ } \\
\text { GINA }\end{array}$ & Yes \\
\hline $\begin{array}{l}\text { Sollander } \\
\text { et al. } 2020^{81}\end{array}$ & $\begin{array}{l}\text { OR of ADD/ } \\
\text { ADHD symptoms } \\
\text { in children with } \\
\text { atopic dermatitis }\end{array}$ & $\begin{array}{l}\text { Cross- } \\
\text { sectional }\end{array}$ & $\begin{array}{l}\text { Uppsala, } \\
\text { Sweden }\end{array}$ & $\begin{array}{l}\text { Not } \\
\text { reported }\end{array}$ & $4451 / 397 / 4054$ & $\begin{array}{l}3-5 \text { years } \\
(4.34) / 50.42 \%\end{array}$ & SDQ & $\begin{array}{l}\text { Asthma/ } \\
\text { question- } \\
\text { naire }\end{array}$ & Yes \\
\hline $\begin{array}{l}\text { Wan et al. } \\
2020^{82}\end{array}$ & $\begin{array}{l}\text { OR of significant } \\
\text { ADD/ADHD } \\
\text { symptoms in chil- } \\
\text { dren with atopic } \\
\text { dermatitis }^{*}\end{array}$ & $\begin{array}{l}\text { Cross- } \\
\text { sectional }\end{array}$ & US & $\begin{array}{l}\text { White } \\
(66.6 \%) \\
\text { Black } \\
(20.9 \%) \\
\text { Indian } \\
(0.9 \%) \text { Asian } \\
(5.3 \%) \\
\text { Multiracial } \\
(6.3 \%)\end{array}$ & $57,726,856 / 6,807,687 / 50,919,169$ & $(10.08) / 48.6 \%$ & SDQ & $\begin{array}{l}\text { Atopic } \\
\text { dermatitis/ } \\
\text { question- } \\
\text { naire }\end{array}$ & Yes \\
\hline
\end{tabular}




\begin{tabular}{|c|c|c|c|c|c|c|c|c|c|}
\hline Study name & $\begin{array}{l}\text { Outcome }(95 \% \\
\text { CI) }\end{array}$ & $\begin{array}{l}\text { Study } \\
\text { design }\end{array}$ & $\begin{array}{l}\text { Country/ } \\
\text { City }\end{array}$ & Ethnicity & $\begin{array}{l}\text { Number of participants/ } \\
\text { Exposed group/Unexposed } \\
\text { group }\end{array}$ & $\begin{array}{l}\text { Age (mean of the } \\
\text { exposed group)/sex } \\
\text { (male, \%) }\end{array}$ & $\begin{array}{l}\text { Diagnostic } \\
\text { criteria for } \\
\text { ADHD }\end{array}$ & \begin{tabular}{|l|} 
Atopic \\
disease/ \\
method \\
used for \\
assessing \\
atopic \\
diseases
\end{tabular} & $\begin{array}{l}\text { Adjustment } \\
\text { for } \\
\text { confounding } \\
\text { factors }\end{array}$ \\
\hline $\begin{array}{l}\text { Berzosa- } \\
\text { Grande et al. } \\
2021^{19}\end{array}$ & $\begin{array}{l}\text { Continuous data } \\
\text { for } \mathrm{IN}^{*}\end{array}$ & $\begin{array}{l}\text { Cross- } \\
\text { sectional }\end{array}$ & $\begin{array}{l}\text { Madrid, } \\
\text { Spain }\end{array}$ & $\begin{array}{l}\text { Not } \\
\text { reported }\end{array}$ & $366 / 194 / 172$ & $\begin{array}{l}6-11 \text { years } \\
(8.68) / 52.7 \%\end{array}$ & CBCL & $\begin{array}{l}\text { Asthma, } \\
\text { atopic } \\
\text { dermatitis, } \\
\text { allergic } \\
\text { rhinitis/ } \\
\text { question- } \\
\text { naire }\end{array}$ & No \\
\hline $\begin{array}{l}\text { Chai et al. } \\
2021^{83}\end{array}$ & $\begin{array}{l}\text { OR of comorbid- } \\
\text { ity of ADHD and } \\
\text { Asthma* }\end{array}$ & $\begin{array}{l}\text { Cross- } \\
\text { sectional }\end{array}$ & Canada & $\begin{array}{l}\text { Not } \\
\text { reported }\end{array}$ & $768,460 / 28,729 / 158,064$ & $(8.7) / 45.8 \%$ & ICD-10 & $\begin{array}{l}\text { Asthma/ } \\
\text { clinical } \\
\text { physician's } \\
\text { diagnosis }\end{array}$ & No \\
\hline $\begin{array}{l}\text { Galéra et al. } \\
2021^{84}\end{array}$ & $\begin{array}{l}\text { Continuous } \\
\text { data for ADHD } \\
\text { symptoms }\end{array}$ & Cohort & Canada & $\begin{array}{l}\text { Not } \\
\text { reported }\end{array}$ & 2120 & $\begin{array}{l}1.5,2.5,3.5,4.5,5 \\
6,7,8,10,13,15 \\
17 \text { years } / 50.9 \%\end{array}$ & $\begin{array}{l}\text { Behavioral } \\
\text { ratings of } \\
\text { ADHD } \\
\text { symptoms }\end{array}$ & $\begin{array}{l}\text { Asthma, } \\
\text { eczema/ } \\
\text { Code of } \\
\text { database } \\
\text { (Quebec } \\
\text { Longitudi- } \\
\text { nal Study } \\
\text { of Child } \\
\text { Develop- } \\
\text { ment } \\
(\text { QLSCD)) }\end{array}$ & Yes \\
\hline $\begin{array}{l}\text { Hou et al. } \\
2021^{85}\end{array}$ & $\begin{array}{l}\text { OR of ADD/ } \\
\text { ADHD in chil- } \\
\text { dren with atopic } \\
\text { dermatitis }^{*}\end{array}$ & $\begin{array}{l}\text { Cross- } \\
\text { sectional }\end{array}$ & US & $\begin{array}{l}\text { Not } \\
\text { reported }\end{array}$ & $228,898 / 23,353 / 205,545$ & $<17$ years $/ 50.2 \%$ & database & $\begin{array}{l}\text { Atopic } \\
\text { dermatitis/ } \\
\text { question- } \\
\text { naire }\end{array}$ & Yes \\
\hline $\begin{array}{l}\text { Keller et al. } \\
2021^{86}\end{array}$ & $\begin{array}{l}\text { Association } \\
\text { between asthma, } \\
\text { atopic dermatitis* } \\
\text { and ADHD } \\
\text { symptoms }\end{array}$ & $\begin{array}{l}\text { Cross- } \\
\text { sectional }\end{array}$ & $\begin{array}{l}\text { Leipzig, } \\
\text { Germany }\end{array}$ & $\begin{array}{l}\text { Not } \\
\text { reported }\end{array}$ & 1764 & $\begin{array}{l}2.53- \\
10.49 \text { years }(6.1) / 53.1 \%\end{array}$ & SDQ & $\begin{array}{l}\text { Asthma, } \\
\text { atopic } \\
\text { dermatitis/ } \\
\text { question- } \\
\text { naire } \\
\end{array}$ & No \\
\hline $\begin{array}{l}\text { Özyurt et al. } \\
2021^{87}\end{array}$ & $\begin{array}{l}\text { Continuous } \\
\text { data for ADHD } \\
\text { symptoms }^{*}\end{array}$ & $\begin{array}{l}\text { Cross- } \\
\text { sectional }\end{array}$ & $\begin{array}{l}\text { Izmir, } \\
\text { Turkey }\end{array}$ & $\begin{array}{l}\text { Not } \\
\text { reported }\end{array}$ & $121 / 61 / 60$ & $\begin{array}{l}12-18 \text { years } \\
(14.27) / 47.54 \%\end{array}$ & SDQ & $\begin{array}{l}\text { Asthma/ } \\
\text { GINA } \\
(2017) \\
\end{array}$ & No \\
\hline $\begin{array}{l}\text { Rajhans } \\
\text { et al. } 2021^{88}\end{array}$ & $\begin{array}{l}\text { Continuous data } \\
\text { for IN*}\end{array}$ & $\begin{array}{l}\text { Cross- } \\
\text { sectional }\end{array}$ & North India & $\begin{array}{l}\text { Not } \\
\text { reported }\end{array}$ & $60 / 30 / 30$ & $8-15$ years $(11.92) / 70 \%$ & $\begin{array}{l}\text { M.I.N.I. } \\
\text { KID, CBCL }\end{array}$ & $\begin{array}{l}\text { Asthma/ } \\
\text { GINA } \\
(2015)\end{array}$ & Yes \\
\hline $\begin{array}{l}\text { Vittrup et al. } \\
2021^{89}\end{array}$ & $\begin{array}{l}\text { HR of ADHD } \\
\text { in children with } \\
\text { atopic dermatitis* }\end{array}$ & Cohort & Denmark & $\begin{array}{l}\text { Not } \\
\text { reported }\end{array}$ & $157,113 / 14,283 / 142,830$ & $0-18$ years $/ 57 \%$ & $\begin{array}{l}\text { Codes of } \\
\text { database }\end{array}$ & $\begin{array}{l}\text { Atopic } \\
\text { dermatitis/ } \\
\text { clinical } \\
\text { physician's } \\
\text { diagnosis }\end{array}$ & Yes \\
\hline $\begin{array}{l}\text { Yüksel et al. } \\
2021^{90}\end{array}$ & $\begin{array}{l}\text { Continuous data } \\
\text { for } \mathrm{HI}^{*} \text { and IN in } \\
\text { ADHD children } \\
\text { with or without } \\
\text { asthma }\end{array}$ & $\begin{array}{l}\text { Cross- } \\
\text { sectional }\end{array}$ & $\begin{array}{l}\text { Izmir, } \\
\text { Turkey }\end{array}$ & $\begin{array}{l}\text { Not } \\
\text { reported }\end{array}$ & $355 / 91 / 264$ & $2-6$ years & $\begin{array}{l}\text { ADHD } \\
\text { Rating } \\
\text { Scale-IV, } \\
\text { K-SADS-PL }\end{array}$ & $\begin{array}{l}\text { Asthma/ } \\
\text { question- } \\
\text { naire }\end{array}$ & Yes \\
\hline
\end{tabular}

Table 1. Studies examining the relationship between atopic diseases and hyperactivity in the qualitative analysis. ADHD, Attention-Deficit/Hyperactivity Disorder; OR, Odds Ratio; HI, Hyperactivity/Impulsivity; IA, Inattention; DSM-IV, Diagnostic and Statistical Manual of Mental Disorders; GINA 2006, Global Initiative for Asthma, 2006; CBCL, Child Behavior Checklist; ARIA, Allergic Rhinitis and its Impact on Asthma; MAST, multiple-antigen simultaneous test; ECI-4, The Early Childhood Inventory-4; ADD, Attention deficit disorder; SDQ, Strengths and Difficulties Questionnaire; ICD-10, International Classification of Diseases, Tenth Revision; SCORAD, SCORing Atopic Dermatitis; ISAAC, International Study of Asthma and Allergies in Childhood; M.I.N.I. KID, Mini-International Neuropsychiatric Interview for Children and Adolescents; K-SADS-PL; Schedule for Affective Disorders and Schizophrenia for School-Aged Children-Present and Lifetime Version (C) Calculated from the available information in the article. ${ }^{\star}$ Statistically significant.

eight studies examined treatment modalities for atopic diseases; however, additional randomized controlled trials are required to obtain more consistent results and causal relationships. The included studies evaluated several confounding factors such as age, sex, and atopic disease severity. Additional detailed information is provided in Table S1 in the Supplementary Materials.

Quantitative review and meta-analysis of cross-sectional studies. Sixteen studies including 25,337 participants examined the relationship between at least one type of atopic disease and the severity of total ADHD symptoms, hyperactivity/impulsivity or inattention, by including unexposed groups consisting of participants without atopic diseases. Table 2 lists the characteristics of included studies. Of the included studies, all studies were cross-sectional designs. 


\begin{tabular}{|c|c|c|c|c|c|c|c|c|}
\hline Study name & Country & Study design & $\begin{array}{l}\text { Number of } \\
\text { participants/ } \\
\text { Exposed group/ } \\
\text { unexposed group }\end{array}$ & $\begin{array}{l}\text { Age (mean of the } \\
\text { exposed group)/ } \\
\text { sex (male, \%) }\end{array}$ & $\begin{array}{l}\text { Diagnostic } \\
\text { criteria for } \\
\text { ADHD }\end{array}$ & $\begin{array}{l}\text { Atopic disease/ } \\
\text { method used for } \\
\text { assessing atopic } \\
\text { diseases }\end{array}$ & Outcome scale & $\begin{array}{l}\text { Overall risk of } \\
\text { bias }\end{array}$ \\
\hline Yuksel et al. $2008^{58}$ & Turkey & Cross-sectional & $100 / 62 / 38$ & $\begin{array}{l}7-12 \text { years } \\
(9.2) / 57 \%\end{array}$ & DSM-IV & $\begin{array}{l}\text { Asthma/physi- } \\
\text { cian's diagnosis } \\
\text { (GINA 2006) }\end{array}$ & $\begin{array}{l}\text { Conner's Parent } \\
\text { Rating Scale }\end{array}$ & Included \\
\hline $\begin{array}{l}\text { Camfferman et al. } \\
2010^{26}\end{array}$ & Australia & Cross-sectional & $107 / 77 / 30$ & $\begin{array}{l}6-16 \text { years } \\
(9.9) / 46.72 \%\end{array}$ & $\begin{array}{l}\text { Conner's Parent } \\
\text { Rating Scale- } \\
\text { Revised }\end{array}$ & $\begin{array}{l}\text { Atopic dermatitis/ } \\
\text { physician's diagno- } \\
\text { sis (Hanifin and } \\
\text { Rajka criteria) }\end{array}$ & $\begin{array}{l}\text { Conner's Parent } \\
\text { Rating Scale }\end{array}$ & Included \\
\hline Lee et al. $2014^{62}$ & Republic of Korea & Cross-sectional & $160 / 87 / 73$ & $\begin{array}{l}\text { 6-13 years } \\
(8.49) / 39.4 \%\end{array}$ & DSM-IV & $\begin{array}{l}\text { Allergic rhinitis/ } \\
\text { physician’s } \\
\text { diagnosis (ARIA } \\
\text { guideline) }\end{array}$ & ARS & Included \\
\hline Yang et al. $2014^{63}$ & Taiwan & Cross-sectional & $122 / 93 / 29$ & $\begin{array}{l}\text { 6-14 years } \\
(10.78) / 52.2 \%\end{array}$ & DSM-IV-TR & $\begin{array}{l}\text { Allergic rhinitis/ } \\
\text { physician's } \\
\text { diagnosis (ARIA } \\
\text { 2008) + IgE test } \\
\text { (MAST or Phadi- } \\
\text { atop tests) }\end{array}$ & SNAP-IV (parent) & Included \\
\hline Catal et al. $2016^{64}$ & Turkey & Cross-sectional & $154 / 80 / 74$ & 3-5 years & ECI-4 & $\begin{array}{l}\text { Atopic dermatitis/ } \\
\text { physician's diagno- } \\
\text { sis (Hanifin and } \\
\text { Rajka criteria) }\end{array}$ & ECI-4 & Included \\
\hline $\begin{array}{l}\text { Hammer-Helmich } \\
\text { et al. } 2016^{65}\end{array}$ & Denmark & Cross-sectional & $9036 / 2433 / 6603$ & $\begin{array}{l}\text { 3-15 years } \\
\text { (NA)/49.5\% }\end{array}$ & SDQ & $\begin{array}{l}\text { Eczema, asthma, } \\
\text { and hay fever/ } \\
\text { Questionnaire }\end{array}$ & SDQ & Included \\
\hline Yang et al. $2016^{67}$ & Taiwan & Case-control & $99 / 68 / 31$ & \begin{tabular}{|l}
$6-14$ years \\
$(9.25) / 82.4 \%$
\end{tabular} & DSM-IV & $\begin{array}{l}\text { Allergic rhinitis/ } \\
\text { Physician's } \\
\text { diagnosis + IgE } \\
\text { test (MAST or } \\
\text { Phadiatop tests) }\end{array}$ & SNAP-IV (parent) & Included \\
\hline Feng et al. $2017^{68}$ & China & Cross-sectional & $643 / 248 / 320$ & $\begin{array}{l}6-12 \text { years } \\
(9.25) / 51.9 \%\end{array}$ & DSM-IV & $\begin{array}{l}\text { Allergic rhinitis/ } \\
\text { Physician's } \\
\text { diagnosis (ARIA } \\
\text { 2008)+skin prick } \\
\text { test }\end{array}$ & SNAP-IV & Included \\
\hline $\begin{array}{l}\text { Kuniyoshi et al. } \\
2018^{70}\end{array}$ & Japan & Cross-sectional & $9954 / 1641 / 8313$ & $\begin{array}{l}7-14 \text { years } \\
(10.42) / 49.9 \%\end{array}$ & SDQ & $\begin{array}{l}\text { Eczema/ISAAC } \\
\text { Eczema Symptom } \\
\text { questionnaire }\end{array}$ & SDQ & Included \\
\hline $\begin{array}{l}\text { Schmitt et al. } \\
2018^{6}\end{array}$ & Germany & Case-control & $89 / 35 / 45$ & $\begin{array}{l}6-12 \text { years } \\
(9.9) / 62.9 \%\end{array}$ & ICD-10 & $\begin{array}{l}\text { Atopic dermatitis/ } \\
\text { Dermatologist's } \\
\text { diagnosis (UK } \\
\text { Working Party } \\
\text { Criteria) }\end{array}$ & FBB-HKS & Included \\
\hline Feng et al. $2020^{74}$ & China & Cross-sectional & $273 / 89 / 184$ & $\begin{array}{l}6-12 \text { years } \\
(8.3) / 62.63 \%\end{array}$ & SNAP-IV & $\begin{array}{l}\text { Atopic dermatitis/ } \\
\text { Dermatologist's } \\
\text { diagnosis (UK } \\
\text { criteria) }\end{array}$ & SNAP-IV & Included \\
\hline Kuo et al. $2020^{41}$ & Taiwan & Cross-sectional & $191 / 109 / 92$ & $(6.59) / 54.44 \%$ & SNAP-IV & $\begin{array}{l}\text { Atopic dermatitis, } \\
\text { asthma, and aller- } \\
\text { gic rhinitis/Physi- } \\
\text { cian's diagnosis }\end{array}$ & SNAP-IV & $\begin{array}{l}\text { "Seek further } \\
\text { information" }\end{array}$ \\
\hline $\begin{array}{l}\text { Minatoya et al. } \\
2020^{79}\end{array}$ & Japan & Cross-sectional & $3862 / 799 / 3063$ & $(5.28) / 49.9 \%$ & SDQ & $\begin{array}{l}\text { Atopic dermatitis/ } \\
\text { ISAAC }\end{array}$ & SDQ & Included \\
\hline $\begin{array}{l}\text { Berzosa-Grande } \\
\text { et al. } 2021^{19}\end{array}$ & Spain & Cross-sectional & $366 / 194 / 172$ & $\begin{array}{l}6-11 \text { years } \\
(8.68) / 52.7 \%\end{array}$ & CBCL & $\begin{array}{l}\text { Asthma, atopic } \\
\text { dermatitis, allergic } \\
\text { rhinitis/question- } \\
\text { naire }\end{array}$ & CBCL & Included \\
\hline $\begin{array}{l}\text { Özyurt et al. } \\
2021^{87}\end{array}$ & Turkey & Cross-sectional & $121 / 61 / 60$ & $\begin{array}{l}12-18 \text { years } \\
(14.27) / 47.54 \%\end{array}$ & SDQ & $\begin{array}{l}\text { Asthma/GINA } \\
(2017)\end{array}$ & SDQ & Included \\
\hline $\begin{array}{l}\text { Rajhans et al. } \\
2021^{88}\end{array}$ & India & Cross-sectional & $60 / 30 / 30$ & $\begin{array}{l}8-15 \text { years } \\
(11.92) / 70 \%\end{array}$ & $\begin{array}{l}\text { M.I.N.I. KID, } \\
\text { CBCL }\end{array}$ & $\begin{array}{l}\text { Asthma/GINA } \\
(2015)\end{array}$ & CBCL & Included \\
\hline
\end{tabular}

Table 2. Studies examining the relationship between atopic diseases and hyperactivity in the meta-analysis. ADHD, Attention-Deficit/Hyperactivity Disorder; DSM-IV, Diagnostic and Statistical Manual of Mental Disorders; GINA 2006, Global Initiative for Asthma, 2006; ARIA, Allergic Rhinitis and its Impact on Asthma; ARS, ADHD Rating Score; MAST, multiple-antigen simultaneous test; ECI-4, The Early Childhood Inventory-4; SNAP-IV, Swanson, Nolan, and Pelham Questionnaire; SDQ, Strengths and Difficulties Questionnaire; CBCL, Child Behavior Checklist; M.I.N.I. KID, Mini-International Neuropsychiatric Interview for Children and Adolescents; ICD-10, International Classification of Diseases, Tenth Revision; FBB-HKS, The German ADHD Rating Scale. 
a

Study

Ozyurt et al., 2021

Minatoya et al., 2021

Kuo et al., 2020

Kuniyoshi et al.

Feng et al., 2020

Catal et al., 2016

Lee et al., 2014

Yuksel et al., 2008

Yang et al., 2014

Schmitt et al., 2017

Yang et al., 2016

Feng et al., 2017

Random-effects

Test for overall effect: $z=5.54(p<0.01)$

Effect size

0.04
0.06
0.22
0.22
0.26
0.31
0.36
0.39
0.41
0.50
0.59
0.62
0.70
0.74

Hammer-Heimich et al., 2016

Heterogeneity: $I^{2}=81 \%, \tau^{2}=0.0359, p<0.0$
Camfferman et al., 2010

C

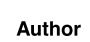

Lee et al., 2014

Yuksel et al., 2008

Kuo et al., 2020

Feng et al., 2020

Schmitt et al., 2017

Camfferman et al.,

Yang et al., 2014

Yang et al., 2016

Feng et al., 2017

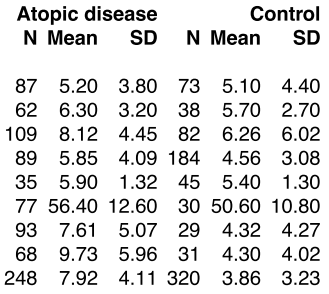

Overall effect

Prediction interval

Heterogeneity: $I^{2}=86 \%[76 \% ; 92 \%], p<0.01$
Standardised Mean

Difference

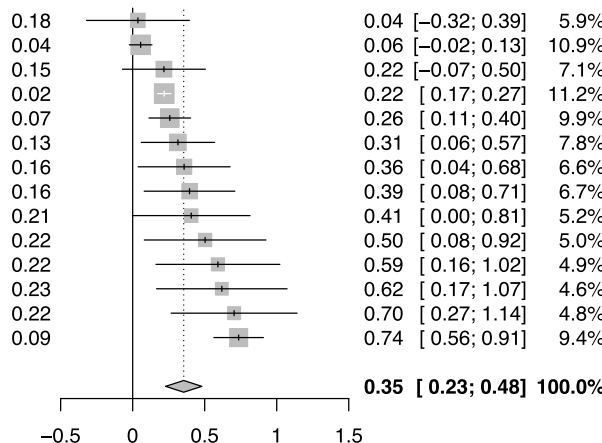

b

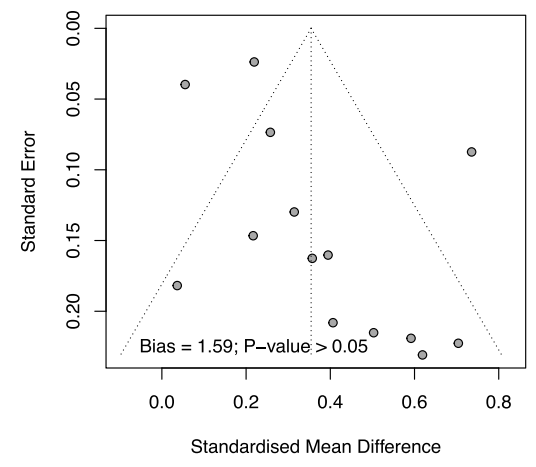

d

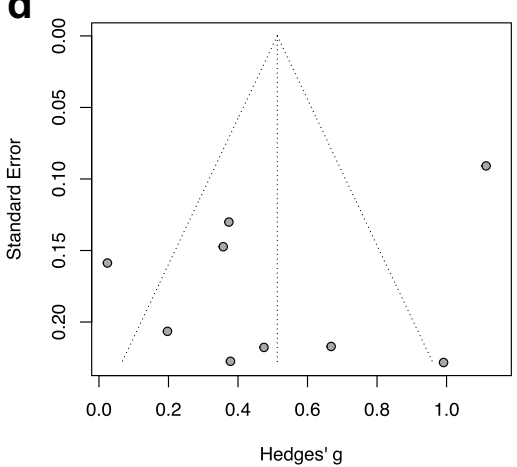

Author

Atopic disease
N Mean SD N Mean SD

$\begin{array}{llllll}109 & 8.48 & 4.19 & 82 & 7.89 & 5.56\end{array}$ $\begin{array}{llllll}89 & 6.61 & 5.02 & 184 & 5.54 & 3.72\end{array}$

Kuo et al., 2020

Berzosa-Grande et al., $2021 \quad 194 \quad 4.693 .78 \quad 172 \quad 3.61 \quad 3.55$

$\begin{array}{lllllll}\text { Yang et al., } 2014 & 93 & 9.98 & 6.35 & 29 & 7.36 & 4.69\end{array}$

Yuksel et al., 2008

Yang et al., 2016

Rajhans et al., 2021

Schmitt et al., 2017

Feng et al., 2017

$\begin{array}{lrllll}62 & 4.20 & 2.30 & 38 & 2.80 & 2.70\end{array}$

$\begin{array}{llllll}68 & 10.95 & 5.68 & 31 & 7.70 & 4.72\end{array}$

$\begin{array}{llllll}30 & 53.13 & 4.99 & 30 & 50.90 & 1.35\end{array}$

$\begin{array}{llllll}35 & 6.00 & 1.38 & 45 & 4.90 & 1.80\end{array}$

$\begin{array}{rrrrrr}248 & 9.79 & 4.67 & 320 & 7.09 & 3.07 \\ 87 & 11.70 & 4.70 & 73 & 7.20 & 5.10\end{array}$

Lee et al., 2014

Overall effect

Prediction interval

Heterogeneity: $I^{2}=66 \%[34 \% ; 83 \%], p<0.01$ f

g $95 \% \mathrm{Cl}$ weight

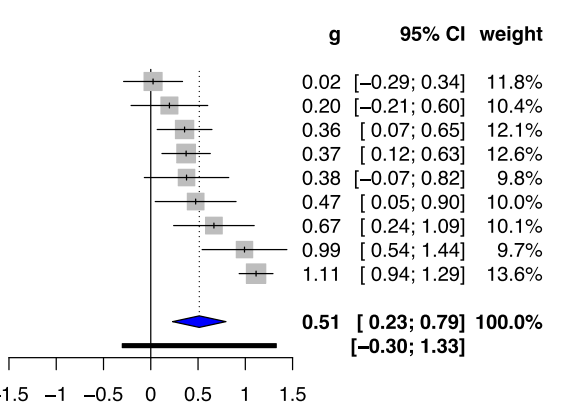

g $95 \% \mathrm{Cl}$ weight

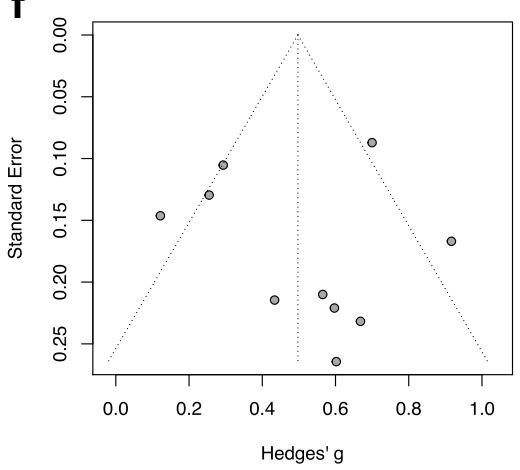

Figure 1. (a) Forest plot of the meta-analysis of the severity of total ADHD symptoms in childhood and the presence of atopic diseases. (b) Funnel plot of the meta-analysis of the severity of total ADHD symptoms in childhood and the presence of atopic diseases. (c) Forest plot of the meta-analysis of the severity of hyperactivity/impulsivity in childhood and the presence of atopic diseases. (d) Funnel plot of the meta-analysis of the severity of hyperactivity/impulsivity in childhood and the presence of atopic diseases. (e) Forest plot of the meta-analysis of the severity of inattention in childhood and the presence of atopic diseases. (f) Funnel plot of the meta-analysis of the severity of inattention in childhood and the presence of atopic diseases. Abbreviations: SD, standard deviation; CI, confidence interval; SMD, standardized mean difference.

Overview of total ADHD symptoms through a meta-analysis. In terms of the outcome of total ADHD symptoms, the overall effect exhibited a statistically significant association with standardized mean difference (SMD) of $0.35,95 \%$ CI of $0.23-0.48$, and $p$ value $<0.01$ (Fig. 1a). We observed considerable heterogeneity among subgroups with an $I^{2}$ value of $81 \%$. The funnel plot displayed no asymmetry $(p>0.05$; Fig. $1 \mathrm{~b})$.

Overview of hyperactivity/impulsivity through a meta-analysis. In terms of the outcome of hyperactivity/impulsivity, the overall effect showed a statistically significant association with atopic diseases, with SMD of $0.51,95 \%$ CI: $0.23-0.79$, and $p$ value of 0.003 (Fig. 1c). Substantial to considerable heterogeneity was noted ( $I^{2}$ value: $86 \%$ ). The funnel plot (Fig. $1 \mathrm{~d}$ ) and Egger's test (Figure S1; $p=0.15$ ) showed no significant publication bias. 
a

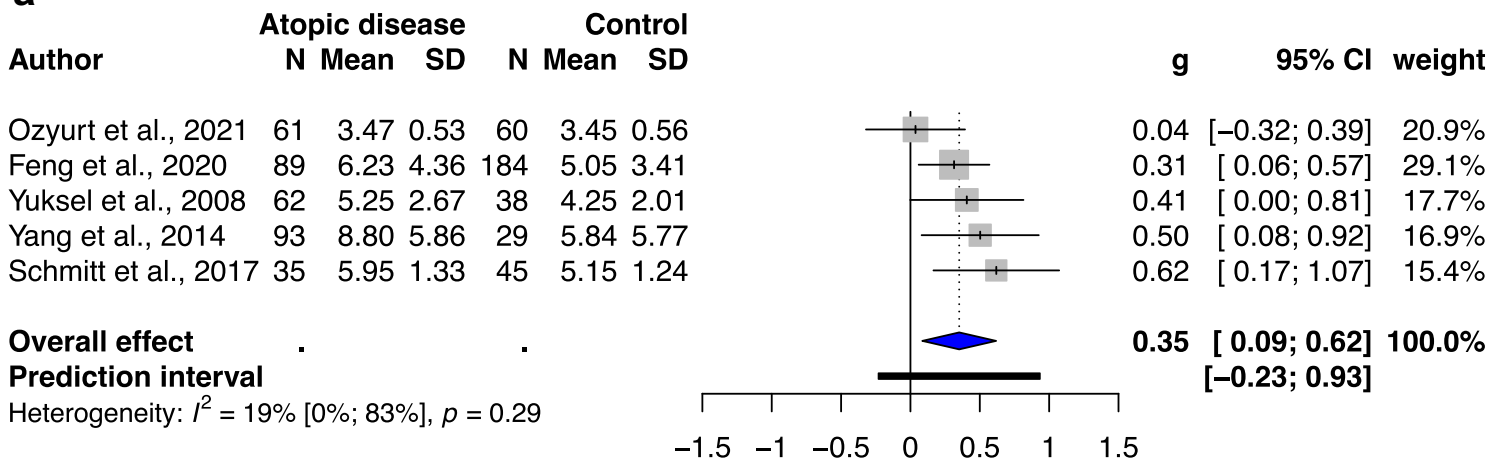

b

\begin{tabular}{lrrrrrr} 
& \multicolumn{2}{c}{ Atopic disease } & \multicolumn{3}{c}{ Control } \\
Author & N Mean & SD & N Mean & SD \\
& & & & & & \\
Yuksel et al., 2008 & 62 & 6.30 & 3.20 & 38 & 5.70 & 2.70 \\
Feng et al., 2020 & 89 & 5.85 & 4.09 & 184 & 4.56 & 3.08 \\
Schmitt et al., 2017 & 35 & 5.90 & 1.32 & 45 & 5.40 & 1.30 \\
Yang et al., 2014 & 93 & 7.61 & 5.07 & 29 & 4.32 & 4.27
\end{tabular}

Overall effect

Prediction interval

Heterogeneity: $I^{2}=0 \%[0 \% ; 85 \%], p=0.47$

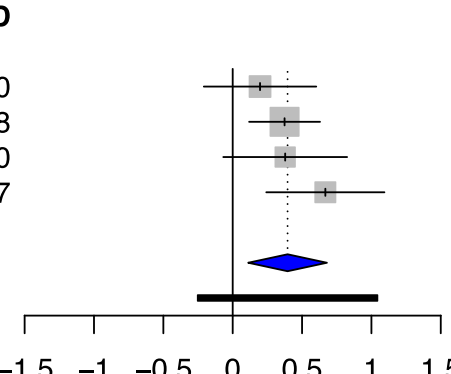

g $95 \% \mathrm{Cl}$ weight

$0.20[-0.21 ; 0.60] \quad 21.7 \%$

0.37 [ $0.12 ; 0.63] \quad 39.4 \%$

$0.38[-0.07 ; 0.82] \quad 18.8 \%$

$0.67[0.24 ; 1.09] \quad 20.1 \%$

$0.40[0.11 ; 0.68] 100.0 \%$ $[-0.25 ; 1.04]$

\section{C}

Author

Feng et al., 2020

Yang et al., 2014

Yuksel et al., 2008

Schmitt et al., 201735

\section{Atopic disease}

N Mean SD

89

$\begin{array}{rrrrrr}89 & 6.61 & 5.02 & 184 & 5.54 & 3.72 \\ 93 & 9.98 & 6.35 & 29 & 7.36 & 4.69 \\ 62 & 4.20 & 2.30 & 38 & 2.80 & 2.70 \\ 35 & 6.00 & 1.38 & 45 & 4.90 & 1.80\end{array}$

\section{Overall effect}

Prediction interval

Heterogeneity: $I^{2}=7 \%[0 \% ; 86 \%], p=0.36$

\section{Control}

N Mean SD

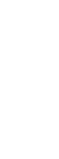

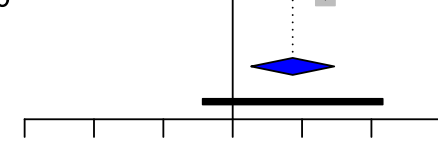

$\begin{array}{lllllll}-1.5 & -1 & -0.5 & 0 & 0.5 & 1 & 1.5\end{array}$

Figure 2. (a) Forest plot of the subgroup analysis of the severity of total ADHD symptoms in childhood and the presence of atopic diseases. (b) Forest plot of the subgroup analysis of the severity of hyperactivity/impulsivity in childhood and the presence of atopic diseases. (c) Forest plot of the subgroup analysis of the severity of inattention in childhood and the presence of atopic diseases. Abbreviations: CI, confidence interval; SMD, standardized mean difference.

Overview of inattention through a meta-analysis. The overall effect of the outcome of inattention showed a significant and positive correlation with atopic diseases, with SMD of 0.5, 95\% CI: 0.32-0.68, and $p$ value of 0.0002 (Fig. 1e). Different from the outcome of hyperactivity/impulsivity, substantial heterogeneity $\left(I^{2}=66 \%\right)$ was observed for the outcome of inattention. The funnel plot (Fig. 1f) and Egger's test (Figure S2; $p=0.88$ ) showed no statistically significant publication bias.

Meta-analysis of ADHD-related behavioral problems in atopic children without previous ADHD diagnosis. Five, four, and four studies included an atopic group without previous ADHD diagnosis and a healthy unexposed group to examine the outcomes of ADHD-related behavioral problems, which indicated total ADHD symptoms, hyperactivity/impulsivity, and inattention, respectively. All studies investigating total ADHD symptoms, hyperactivity/impulsivity, and inattention exhibited a statistically significant association, with SMD of 0.35 (95\% CI: $0.09-0.62$, $p$ value: 0.0212 (Fig. $2 \mathrm{a}$ )), SMD of 0.40 (95\% CI: $0.11-0.68, p$ value: 0.0212 (Fig. 2b)), and SMD of $0.43(95 \%$ CI: $0.13-0.73$, $p$ value: 0.0193 (Fig. 2 c)), respectively. Low heterogeneity was noted in this meta-analysis $\left(I^{2}=0-19 \%\right)$.

Subgroup analysis of different types of atopic diseases. A significant association was observed between atopic dermatitis and total ADHD symptoms (SMD: 0.28, 95\% CI: 0.09-0.47, $p$ value: 0.012 ) and 
a

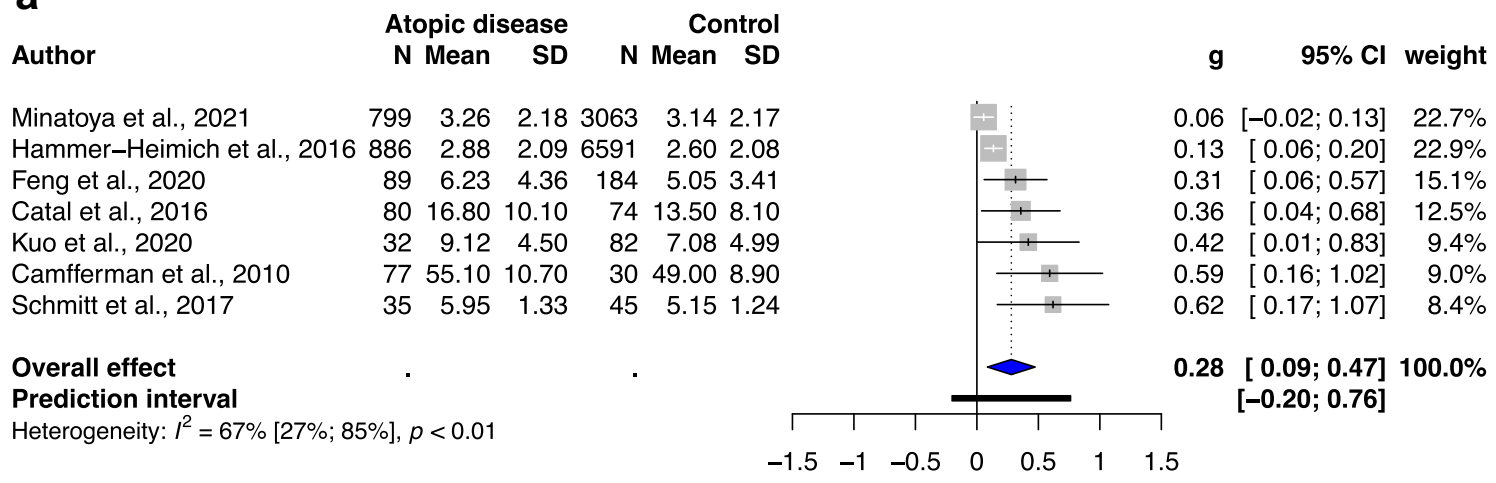

b

Author Atopic disease Control

$\begin{array}{llllllll}\text { Feng et al., } 2020 & 89 & 5.85 & 4.09 & 184 & 4.56 & 3.08\end{array}$

Schmitt et al., $2017 \quad 35 \quad 5.90 \quad 1.32 \quad 45 \quad 5.40 \quad 1.30$

Camfferman et al., $201077 \quad 56.40 \quad 12.60 \quad 30 \quad 50.60 \quad 10.80$

$\begin{array}{lllllll}\text { Kuo et al., } 2020 & 32 & 8.96 & 3.86 & 82 & 6.26 & 6.02\end{array}$

Overall effect

Prediction interval

Heterogeneity: $I^{2}=0 \%[0 \% ; 85 \%], p=0.96$

$0.06[-0.02 ; 0.13] 22.7 \%$

$0.13[0.06 ; 0.20] \quad 22.9 \%$

$0.31[0.06 ; 0.57] \quad 15.1 \%$

$0.36 \quad[0.04 ; 0.68] \quad 12.5 \%$

$0.42[0.01 ; 0.83] \quad 9.4 \%$

$0.59[0.16 ; 1.02] \quad 9.0 \%$

$0.28[0.09 ; 0.47] 100.0 \%$ $[-0.20 ; 0.76]$

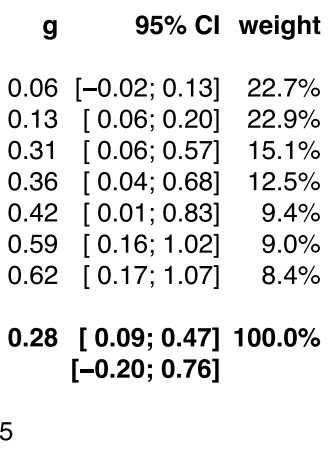

g $95 \% \mathrm{Cl}$ weight

$0.37[0.12 ; 0.63] \quad 48.2 \%$

$0.38[-0.07 ; 0.82] 15.9 \%$

$0.47[0.05 ; 0.90] \quad 17.4 \%$

$0.49[0.07 ; 0.90] \quad 18.5 \%$

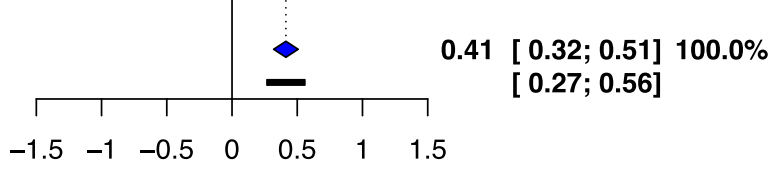

\section{C \\ Author \\ Atopic disease N Mean SD N Mean SD}

Feng et al., 2020

Kuo et al., 2020

Schmitt et al., 201735

Overall effect Prediction interval

Heterogeneity: $l^{2}=22 \%[0 \% ; 92 \%], p=0.28$

$\begin{array}{rrrrr}6.61 & 5.02 & 184 & 5.54 & 3.72 \\ 9.29 & 4.58 & 82 & 7.89 & 5.56 \\ 6.00 & 1.38 & 45 & 4.90 & 1.80\end{array}$

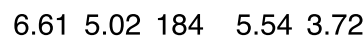
$\begin{array}{lllll}6.00 & 1.38 & 45 & 4.90 & 1.80\end{array}$

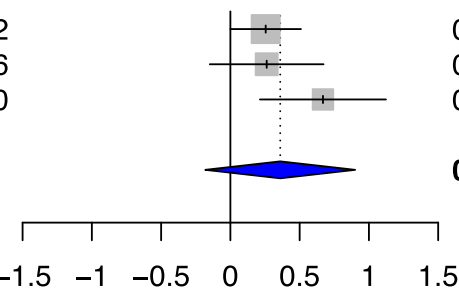

g $\quad 95 \% \mathrm{Cl}$ weight

$0.25[0.00 ; 0.51] \quad 46.6 \%$

$0.26[-0.15 ; 0.67] \quad 28.5 \%$

$0.67[0.21 ; 1.12] \quad 24.9 \%$

$0.36[-0.18 ; 0.90] 100.0 \%$ [-2.22; 2.94]

Figure 3. (a) Forest plot of the subgroup analysis of the severity of total ADHD symptoms in children with atopic dermatitis. (b) Forest plot of the subgroup analysis of the severity of hyperactivity/impulsivity in children with atopic dermatitis. (c) Forest plot of the subgroup analysis of the severity of inattention in children with atopic dermatitis. Abbreviations: $\mathrm{CI}$, confidence interval; SMD, standardized mean difference.

hyperactivity/impulsivity (SMD: $0.41,95 \%$ CI: $0.32-0.51, p$ value: 0.0008 ), with low to moderate heterogeneity $\left(I^{2}=0-67 \%\right.$; Fig. 3). A significant association was noted between allergic rhinitis and total ADHD symptoms (SMD: 0.53 , 95\% CI: $0.26-0.80$, $p$ value: 0.006 ), hyperactivity/impulsivity (SMD: $0.62,95 \%$ CI: $0.05-1.20, p$ value: 0.04 ), and inattention (SMD: $0.56,95 \%$ CI: $0.18-0.94, p$ value: 0.015 ), with moderate to substantial heterogeneity $\left(I^{2}=59-91 \%\right.$; Fig. 4$)$. Furthermore, a significant association was observed between asthma and only total ADHD symptoms (SMD: $0.25,95 \% \mathrm{CI}: 0.07-0.42$, $p$ value: 0.02 ), with low to substantial heterogeneity $\left(I^{2}=0-55 \%\right.$; Fig. 5). The analysis of hyperactivity in children with asthma was not performed due to insufficient studies $(\mathrm{n}=2)$.

As sensitivity analysis, we observed decreased heterogeneity in meta-analysis of ADHD-related behavioral problems in children without previous ADHD diagnosis. However, in subgroups were categorized according to the types of atopic diseases, only the atopic dermatitis and asthma subgroup showed decreased heterogeneity $\left(I^{2}=0-67 \%\right)$, whereas allergic rhinitis subgroup showed low to considerable heterogeneity.

\section{Discussion}

The results of our study indicated that atopic diseases were related to not only increased odds of ADHD but also the severity of ADHD symptoms. Total ADHD symptoms, hyperactivity/impulsivity, and inattention were all significantly associated with atopic diseases. The results of the subgroup analysis indicated that children with subthreshold ADHD in the atopic disease groups had increased severity of ADHD symptoms, including total ADHD symptoms, hyperactivity/impulsivity, and inattention, and atopic dermatitis and allergic rhinitis were significantly associated with ADHD symptoms. 
a

Author

Lee et al., 2014

Yang et al., 2014

Yang et al., 2016

Feng et al., 2017248
Atopic disease

$N$ Mean SD
Control

N Mean SD

Overall effect

Prediction interval

Heterogeneity: $I^{2}=59 \%[0 \% ; 85 \%], p=0.05$

7.084 .99

6.155 .31

5.845 .77

6.005 .61

5.474 .58

$\begin{array}{rrrrrr}95 & 8.22 & 4.08 & 82 & 7.08 & 4.99 \\ 87 & 8.45 & 6.18 & 73 & 6.15 & 5.31 \\ 93 & 8.80 & 5.86 & 29 & 5.84 & 5.77 \\ 68 & 10.34 & 6.33 & 31 & 6.00 & 5.61 \\ 48 & 8.86 & 4.60 & 320 & 5.47 & 4.58\end{array}$

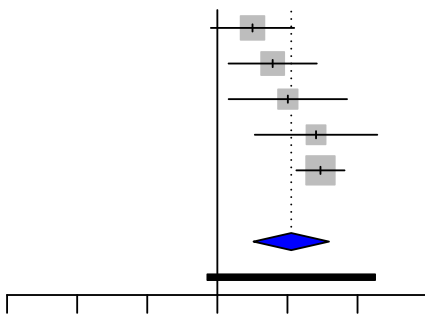

g $\quad 95 \% \mathrm{Cl}$ weight

$0.25[-0.05 ; 0.55] \quad 21.2 \%$

$0.39[0.08 ; 0.71] \quad 20.0 \%$

0.50 [ $0.08 ; 0.92] \quad 14.3 \%$

$0.70[0.27 ; 1.14] \quad 13.7 \%$

$0.74[0.56 ; 0.91] \quad 30.8 \%$

$0.53[0.26 ; 0.80] 100.0 \%$ $[-0.07 ; 1.12]$

b

\begin{tabular}{|c|c|c|c|c|c|}
\hline & \multicolumn{3}{|c|}{ Atopic disease } & & \\
\hline Author & $\mathbf{N}$ & Mean & SD & $\mathbf{N}$ & Mean \\
\hline t al., 2014 & 87 & 5.20 & 3.80 & 73 & 5.1 \\
\hline o et al., 2020 & 95 & 7.93 & 4.34 & 82 & 6.26 \\
\hline ng et al., 2014 & 93 & 7.61 & 5.07 & 29 & 4.32 \\
\hline g et al., 2016 & 68 & 9.73 & 5.96 & 31 & 4.30 \\
\hline ng et al., 2017 & 248 & 7.92 & 4.11 & 320 & 3.86 \\
\hline
\end{tabular}

Control

$\begin{array}{lllllll}-1.5 & -1 & -0.5 & 0 & 0.5 & 1 & 1.5\end{array}$

Overall effect
Prediction interval
Heterogeneity: $I^{2}=91 \%[83 \% ; 96 \%], p<0.01$

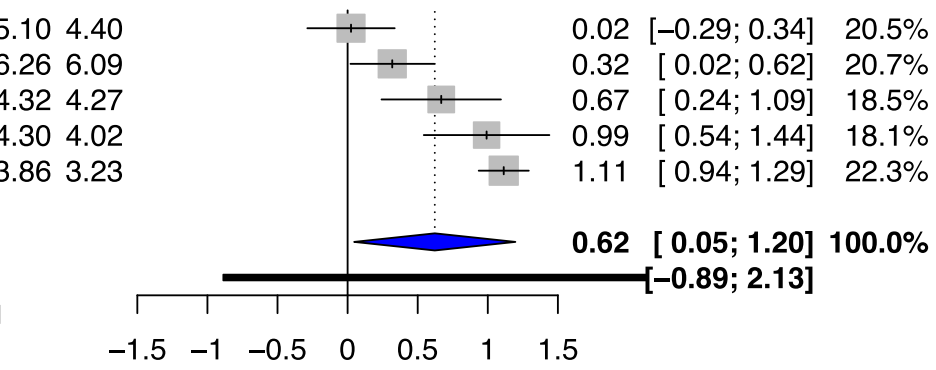

C

\begin{tabular}{lrrrrrr} 
& \multicolumn{2}{c}{ Atopic disease } & & \multicolumn{2}{c}{ Control } \\
Author & N Mean & SD & N Mean & SD \\
& & & & & & \\
Kuo et al., 2020 & 95 & 8.50 & 4.22 & 82 & 7.89 & 5.56 \\
Yang et al., 2014 & 93 & 9.98 & 6.35 & 29 & 7.36 & 4.69 \\
Yang et al., 2016 & 68 & 10.95 & 5.68 & 31 & 7.70 & 4.72 \\
Feng et al., 2017 & 248 & 9.79 & 4.67 & 320 & 7.09 & 3.07 \\
Lee et al., 2014 & 87 & 11.70 & 4.70 & 73 & 7.20 & 5.10
\end{tabular}

\section{Overall effect}

Prediction interval

Heterogeneity: $I^{2}=74 \%[36 \% ; 90 \%], p<0.01$

\section{g $95 \% \mathrm{Cl}$ weight}

$0.32[0.02 ; 0.62] \quad 20.7 \%$

$0.67[0.24 ; 1.09] \quad 18.5 \%$

$0.99[0.54 ; 1.44] \quad 18.1 \%$

$1.11[0.94 ; 1.29] \quad 22.3 \%$

Figure 4. (a) Forest plot of the subgroup analysis of the severity of total ADHD symptoms in children with allergic rhinitis. (b) Forest plot of the subgroup analysis of the severity of hyperactivity/impulsivity in children with allergic rhinitis. (c) Forest plot of the subgroup analysis of the severity of inattention in children with allergic rhinitis. Abbreviations: CI, confidence interval; SMD, standardized mean difference.

Although we included a small number of studies, these studies in total included more than 25,000 participants. In addition, this is the first systematic review and meta-analysis to investigate not only the association of atopic diseases with the severity of ADHD symptoms but also the presentation of ADHD-related behavioral symptoms in atopic children without previous ADHD diagnosis. The results of both the qualitative systematic review and meta-analysis tended to demonstrate higher severity of total ADHD symptoms and inattention in participants with atopic diseases, and the meta-analysis showed a significant association between atopic diseases and hyperactivity-impulsivity severity although qualitative synthesis showed relatively inconsistent outcome. Three studies that adopted the computerized CAT to assess attention deficits also demonstrated similar results, thus supporting our hypothesis. However, our qualitative systematic review and meta-analysis exhibited inconsistency in outcomes and considerable heterogeneity.

We performed a second meta-analysis of ADHD-related behavioral problems in atopic children without previous ADHD diagnosis, which not only consisted of studies with more strict exclusion criteria for diagnosed ADHD, but also reflected on the association between atopic diseases and subthreshold ADHD. The result showed statistical significance with low heterogeneity, indicating that atopic children was associated with subthreshold ADHD, as ADHD-related behavioral problems, despite of lack of diagnosed ADHD. The subgroup analysis of different types of atopic diseases showed that atopic dermatitis was significantly and positively correlated 
a

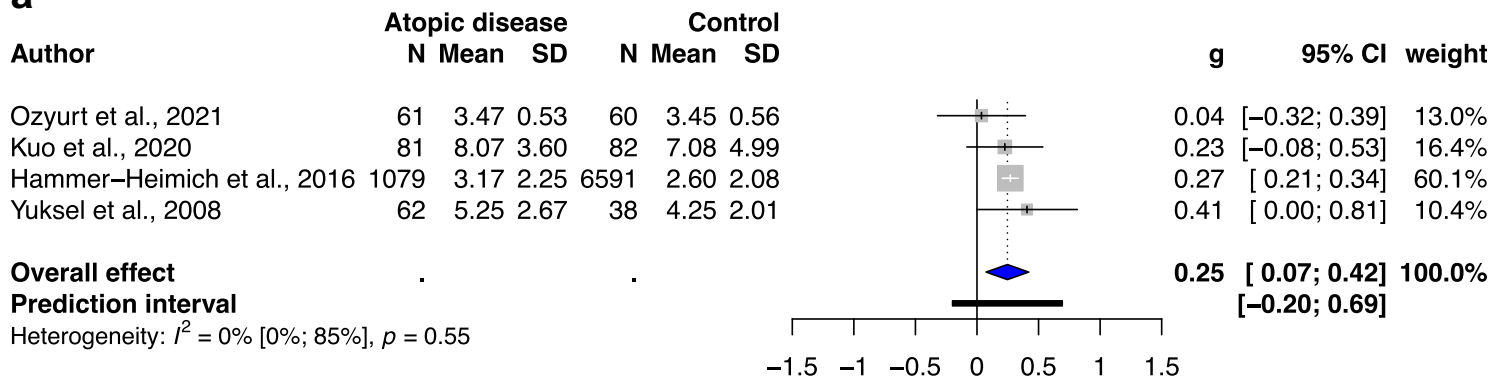

b

\begin{tabular}{ccc} 
& Atopic disease & \multicolumn{2}{c}{ Control } \\
Author & $\mathrm{N}$ Mean SD N Mean SD
\end{tabular}

g $\quad 95 \% \mathrm{Cl}$ weight

$\begin{array}{lllllll}\text { Kuo et al., } 2020 \quad 81 & 8.38 & 3.74 & 82 & 7.89 & 5.56\end{array}$

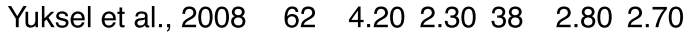

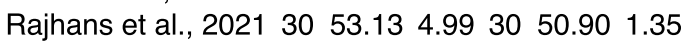
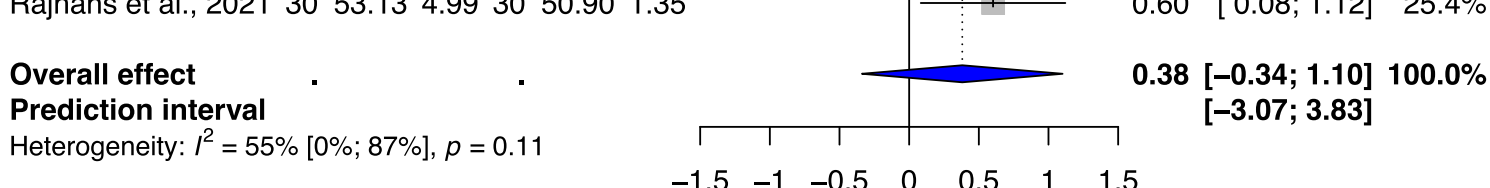

Figure 5. (a) Forest plot of the subgroup analysis of the severity of total ADHD symptoms in children with asthma. (b) Forest plot of the subgroup analysis of the severity of hyperactivity/impulsivity in children with asthma. (c) Forest plot of the subgroup analysis of the severity of inattention in children with asthma. Abbreviations: CI, confidence interval; SMD, standardized mean difference.

with the severity of total ADHD symptoms and hyperactivity/impulsivity, with low to moderate heterogeneity $\left(I^{2}=0-67 \%\right)$. Allergic rhinitis was significantly associated with the severity of total ADHD symptoms, hyperactivity/impulsivity, and inattention, with moderate to considerable heterogeneity $\left(I^{2}=59-91 \%\right)$. Asthma was significantly associated only with the severity of total ADHD symptoms, with low to substantial heterogeneity $\left(I^{2}=0-55 \%\right)$.

This inconsistency in heterogeneity can be attributed to differences in participants' sex and mean age and the inclusion and exclusion criteria of related studies. A previous study reported a higher male to female ratio for hyperactivity but an equal male to female ratio for inattention ${ }^{27}$. This study also indicated that the distribution of sex differences within the severity of symptoms varied between children without ADHD and children with ADHD. The differences in the clinical course of participants can also result in these inconsistencies. Hyperactivity/impulsivity is usually observed from 4 years of age, peaks in severity at approximately 7 to 8 years, and then declines later, whereas inattention is not apparent until 8-9 years of age ${ }^{28}$. In this study, we observed a significantly decreased heterogeneity in the meta-analysis of ADHD-related behavioral problems in atopic children without previously diagnosed ADHD, indicating that inconsistent exclusion criteria for ADHD may have been the source of heterogeneity in our study. Moreover, the lack of standard inclusion and exclusion criteria for not only ADHD but also other neuropsychiatric disorders or developmental delays may be responsible for the high heterogeneity in our study. Furthermore, our results suggested that children with atopic diseases without previous ADHD diagnosis had a stronger association of more severe core symptoms of ADHD than healthy unexposed groups, indicating a more consistent result for inattention than hyperactivity/impulsivity, with remarkably decreased heterogeneity.

We also observed low to considerable heterogeneity in the subgroup analysis of different types of atopic diseases. Asthma had a significant association only with the severity of total ADHD symptoms, not with inattention. Considering that previous studies have reported that children with asthma had an increased risk of developing severe ADHD symptoms ${ }^{18,22,29}$, this result may be attributed to the small number of included studies $(n=3)$. The results of the subgroup analysis of different types of atopic diseases indicated that all atopic diseases were significantly associated with the severity of total ADHD symptoms; however, inconsistent heterogeneity was observed in the association between atopic diseases and the severity of hyperactivity/impulsivity and inattention. These findings suggest that different types of atopic diseases may have distinct interactions with ADHD symptoms, although our results demonstrated that any type of atopic disease was associated with the severity of ADHD symptoms.

Although the causal relationship and mechanism underlying the association between atopic diseases and ADHD remain unclear, many studies have provided several hypotheses ${ }^{30}$ ) (Figure S3). One of the more commonly accepted hypotheses is that atopic diseases induce inflammation, including Th1, Th2, and Th17 immune responses, which lead to the downstream hypersecretion of $\operatorname{IgE}^{31-33}$ (Figure S4). These inflammatory factors may affect the neuroactivity of the prefrontal cortex (PFC) and anterior cingulate cortex (ACC) that have been reported to be strongly associated with ADHD pathology ${ }^{34-36}$. This process affects the maturation of the PFC 
and ACC, which usually occurs in early life when the brain is undergoing profound changes and is important for the development of cognitive function ${ }^{37}$. In addition to the direct effect, cytokines may indirectly affect these brain areas by disturbing the hypothalamic-pituitary-adrenal axis (HPA) axis ${ }^{38}$, altering the central metabolism of neurotransmitters including norepinephrine and dopamine $e^{35,39}$.

In addition to neuroimmunological pathways, psychological mechanisms should also be considered. Atopic diseases cause psychological stress in not only patients but also their main caregivers since early childhood when the onset of atopic diseases occurs ${ }^{40,41}$. An unsatisfactory parent-child relationship characterized by overprotection, anxiety, low support, and poor sleep quality can lead to a decline in psychosocial and cognitive performance due to tiredness; emotional problems were reported to be associated with negative outcomes in a previous study ${ }^{42}$. Although the relationship between these effects and brain development in early life has not yet been well studied, stress in early childhood is believed to affect the balance between neurotransmitters and neuroendocrine systems, including norepinephrine, dopamine, and the HPA axis ${ }^{38,43}$. This eventually resulted in increased vulnerability to psychological diseases, such as ADHD, because of altered neuropsychologic pathways leading to unsuccessful brain development and maturation ${ }^{44}$.

An increased risk of the development and progression of atopic diseases was observed in children with ADHD because atopic diseases are attributed to stress ${ }^{45}$. Although these mechanisms were obscure, studies have found that stress may induce deficits in skin barriers, and a similar inflammatory cascade with atopy-associated immune responses was observed in patients with atopic diseases; some patients exhibited an increased tendency to exhibit Th1 responses ${ }^{46}$. These findings indicate that stress can exacerbate atopic diseases. ADHD was reported to be related to psychosocial stress because poor family support, school performance, and peer relationships can all be the sources of stress ${ }^{47}$. Therefore, ADHD-related stress can exacerbate atopic diseases. Recently, genetic factors and prenatal stress have been indicated as common risk factors for atopic diseases and ADHD. Although few studies have evaluated genetic susceptibility and other associated interaction factors leading to epigenetic reactions ${ }^{48,49}$, a twin study supported the hypothesis that common genetic factors between atopy and ADHD exist ${ }^{50}$. Moreover, studies reporting a relationship between maternal stress and symptoms of atopic diseases and ADHD have indicated that dysregulation of the HPA axis results in delayed brain development and a shift in Th1/Th2 balance, resulting in an atopic disease-prone immune profile ${ }^{51,52}$

Previous systematic reviews and meta-analyses had discussed about increased risk of diagnosed ADHD in atopic group; however, the evidence of whether atopic diseases associated with increased severity of ADHD symptoms in participants with subthreshold ADHD remained unclear. This association could be observed in the meta-analysis of ADHD-related behavioral problems in atopic children without previously diagnosed ADHD in the present study, provided an evidence that atopic diseases are associated with "spectrum of ADHD symptom severity", the term which we quoted following a previous study to explain how atopic diseases associated with ADHD symptoms ${ }^{53}$, and this association existed in subthreshold ADHD group in our result as well.

Limitations. This study has several limitations that should be considered. First, although more than 25,000 participants were included, the outcome of hyperactivity was examined in only 1700 participants, which may reduce the strength of evidence. Second, Atopic diseases were diagnosed based on physicians' decisions or by using some questionnaires in the included studies; these might have caused heterogeneity. Third, we chose to combine scores of hyperactivity/impulsivity and inattention as the primary outcome, and this method has been used in several studies ${ }^{54}$. However, potential systematic errors may still occur. Forth, differences in exclusion criteria, including the exclusion of patients with ADHD and other neuropsychiatric disorders and development problems, caused significant heterogeneity in our study. Finally, some studies included children with multiple atopic diseases, which may be potential confounding factors in our study.

To minimize the effect of those limitations, we conducted sensitivity analysis and subgroup analysis to assess the effects of potential confounding factors, namely different types of atopic diseases and exclusion criteria for previous diagnosed ADHD and other neuropsychiatric disorders; this considerably reduced heterogeneity in sensitivity analysis and most subgroup analyses. Therefore, standardized and more strictly defined exclusion and inclusion criteria should be included in future studies. Moreover, additional studies investigating the relationship between different types of atopic diseases and ADHD symptoms should be conducted because we could not conduct a complete subgroup analysis of different atopic diseases owing to the limited number of studies.

\section{Conclusion}

Our study results indicated that atopic diseases not only increased the odds of ADHD but also were associated with more severe core symptoms of ADHD. We observed increased severity of ADHD-related behavioral symptoms in children with atopic diseases without previously diagnosed ADHD, indicating that atopic diseases may also associated with spectrum of ADHD symptom severity in participants with subthreshold ADHD, which was never been investigated in previous researches. According to the results of this study, while treating children with ADHD, clinicians should consider the possibility of comorbid atopic diseases. On the other hand, the comorbidity of ADHD should be considered when treating children with atopic diseases. Moreover, clinicians should be aware of the increased ADHD-related behavioral symptoms in children with atopic diseases. Additional studies including more strictly defined criteria, studies investigating the mechanism underlying this association, and randomized controlled trials of related therapeutic strategies should be conducted. 


\section{Methods}

This systematic review and meta-analysis was conducted in accordance with the Preferred Reporting Items for Systematic Reviews and Meta-Analyses (PRISMA) guidelines ${ }^{55}$. Related checklists are provided in the Supplementary Materials. Two researchers searched for and pooled observational studies examining atopic diseases and ADHD symptom severity to examine the association between them.

Eligibility criteria for study selection. To reduce selection bias, we defined eligibility criteria before the inclusion of studies. We included the following studies: (1) observational studies investigating the association between one of the three major types of atopic diseases (i.e., asthma, eczema, and allergic rhinitis) and the severity of ADHD symptoms, irrespective of whether they included patients with ADHD in the exposed group; (2) studies recruiting children and adolescents; (3) studies including any of the three atopic diseases as the exposure variable and ADHD symptom severity (scores of a behavior rating scale) as the outcome variable; (4) studies investigating the severity of ADHD symptoms in patients with at least one type of atopic disease by using any type of assessment method; (5) studies including a matched or an unmatched unexposed group for comparison with the exposed group; and (6) cross-sectional, case-control, or cohort studies. The following studies were excluded: (1) studies that did not provide adequate information regarding the relationship between atopic diseases and ADHD symptom severity, including the crude data of exposed cases and the outcome of the association between at least one type of atopic disease and the severity of hyperactivity/impulsivity, inattention, and/or total ADHD symptoms (hyperactivity/impulsivity + inattention) determined on the basis of the scores of a behavior rating scale; (2) studies including only outcomes measured after interventions or those not including a separate outcome of total ADHD symptoms, hyperactivity/impulsivity, and inattention; (3) studies using the same database in different published articles (studies with longer follow-up periods and higher quality, which were classified as included (see 'Table S2 in the Supplementary Materials') were included); and (4) studies whose full texts were not available or those for which only abstracts or editorial materials (i.e., comments, responses, and letters without original data) were available.

Search strategy and study selection. Two authors performed a literature search on PubMed (Medline), Embase, and Psycinfo for studies published up to December 18, 2021. The following search terms were combined and adjusted to fulfill the demand of the database.

"Atopic eczema" [MeSH] OR "atopic dermatitis" [MeSH] OR "asthma” [MeSH] OR "allergic rhinitis" [MeSH] "ADHD” [MeSH] OR "attention deficit and hyperactivity disorder" [MeSH] OR "hyperactivity" [MeSH] OR “inattention" [MeSH] OR "attention deficit” [Mesh] OR “impulsivity" [Mesh] OR "mental health" [MeSH] OR "behavior problem" [MeSH]

"Preschool children" OR "preschooler" OR "children” OR “adolescent” OR “toddler"

A three-step screening strategy was adopted. In the title screening step, studies that met the inclusion criteria and were not letters or replies were included. In the abstract screening step, studies that were original research and included relevant unexposed groups were included. In the full-text screening step, studies for which full texts and data were available and those that did not meet the exclusion criteria were finally included. A third author resolved any disagreement between the two authors in terms of the eligibility and inclusion of studies through discussion.

Data extraction. By using a customized data form, the first author extracted the following information from eligible studies: study title, study design, study characteristics (country, composition of participants, and outcome assessment scales used), and assessment methods and criteria for diagnosing atopic diseases and ADHD. Subsequently, the second author reviewed the extracted data. Any disagreement regarding data extraction was resolved by the third author through discussion. If required, data were calculated from the available data of included studies (see 'Data abstraction for the meta-analysis'). The main outcome variables were the scores of the behavior rating scales used to evaluate hyperactivity/impulsivity and inattention in the atopic disease and unexposed groups. Although the included studies used different outcome scales, they were all based on the Diagnostic and Statistical Manual of Mental Disorders, 4th Edition (DSM-IV) criteria. In addition, to minimize the potential confounding effects, we analyzed standardized data. Because of the limited availability of studies, we combined the three types of atopic diseases in the meta-analysis and conducted a subgroup analysis. The authors individually recorded abstracted data in Microsoft Excel.

Data abstraction for the meta-analysis. The relationship between one of the three main types of atopic diseases with the severity of hyperactivity/impulsivity, inattention, or total ADHD symptoms was independently investigated. To exclude confounding factors, any study including outcomes measured after interventions were excluded. For each study, the relative weight of outcome data to the whole data set was calculated using the generic inverse variance method along with the $95 \%$ confidence interval (95\% CI) by using Rstudio software, Version 1.2.5042 Rstudio ${ }^{56}$. The random effects model with the Hartung-Knapp-Sidik-Jonkman method was used to determine the estimated variance for pooling effect sizes in our meta-analysis, with standardized mean difference as effect size. The $I^{2}$ value was calculated to assess statistical heterogeneity, and this value represents the total variance of pooled data explained by the heterogeneity. According to the Cochrane handbook for systematic reviews of interventions, the $I^{2}$ values of $0-40 \%, 30-60 \%, 50-90 \%$, and $75-100 \%$ indicated low, moderate, substantial, and considerable heterogeneity ${ }^{57}$. We used a funnel plot to assess the potential publication bias. 
For the outcome "severity of total ADHD symptoms," if a study reported ADHD symptoms in "severity of hyperactivity/impulsivity" and "severity of inattention" separately, we calculated weighted mean and pooled standard deviation based on relevant formula before meta-analysis ${ }^{57}$.

For studies including a subgroup analysis, we pooled effect sizes two times. First, we combined subgroups within studies to pool effect sizes and calculated $95 \%$ CIs and standard errors. Subsequently, we included the calculated standard errors into our meta-analysis and pooled effect sizes a second time.

Sensitivity analysis. To assess the strength of the association between atopic diseases and ADHD in different scenarios, we performed a sensitivity analysis by investigating patients with strictly defined exclusion criteria for previous ADHD diagnosis. We included studies researching both exposed and unexposed groups with exclusion criteria for previous ADHD diagnosis for a second meta-analysis, which was mentioned as meta-analysis of ADHD-related behavioral problems in atopic children without previous ADHD diagnosis. Detailed information on the risk of bias assessment could be found in Table S2 in the Supplementary Materials.

Subgroup analysis of different type of atopic diseases. We conducted subgroup analysis of different type of atopic diseases in three outcomes: total ADHD symptoms, hyperactivity, and inattention, using a random effect model. If there were less than 3 studies included in each subgroup, we would not perform analysis.

\section{Data availability}

The datasets generated during and/or analyzed during the current study are available from the corresponding author on reasonable request.

Received: 26 July 2021; Accepted: 9 February 2022

Published online: 01 March 2022

\section{References}

1. Worldwide variation in prevalence of symptoms of asthma, allergic rhinoconjunctivitis, and atopic eczema: ISAAC. The International Study of Asthma and Allergies in Childhood (ISAAC) Steering Committee. Lancet 351, 1225-1232 (1998).

2. Asher, M. I. et al. Worldwide time trends in the prevalence of symptoms of asthma, allergic rhinoconjunctivitis, and eczema in childhood: ISAAC Phases One and Three repeat multicountry cross-sectional surveys. Lancet 368, 733-743. https://doi.org/10. 1016/s0140-6736(06)69283-0 (2006).

3. Schmitt, J. et al. Infant eczema, infant sleeping problems, and mental health at 10 years of age: the prospective birth cohort study LISAplus. Allergy 66, 404-411. https://doi.org/10.1111/j.1398-9995.2010.02487.x (2011).

4. Stanescu, S., Kirby, S. E., Thomas, M., Yardley, L. \& Ainsworth, B. A systematic review of psychological, physical health factors, and quality of life in adult asthma. NPJ Prim Care Respir Med 29, 37. https://doi.org/10.1038/s41533-019-0149-3 (2019).

5. Ferrante, G. \& La Grutta, S. The Burden of Pediatric Asthma. Front Pediatr 6, 186. https://doi.org/10.3389/fped.2018.00186 (2018).

6. Schmitt, J. et al. Increased attention-deficit/hyperactivity symptoms in atopic dermatitis are associated with history of antihistamine use. Allergy 73, 615-626. https://doi.org/10.1111/all.13326 (2018).

7. Hill, M. K., Kheirandish Pishkenari, A., Braunberger, T. L., Armstrong, A. W. \& Dunnick, C. A. Recent trends in disease severity and quality of life instruments for patients with atopic dermatitis: A systematic review. J. Am. Acad. Dermatol. 75, 906-917. https:// doi.org/10.1016/j.jaad.2016.07.002 (2016).

8. O'Connell, E. J. The burden of atopy and asthma in children. Allergy 59(Suppl 78), 7-11. https://doi.org/10.1111/j.1398-9995.2004. 00563.x (2004)

9. Polanczyk, G. V., Willcutt, E. G., Salum, G. A., Kieling, C. \& Rohde, L. A. ADHD prevalence estimates across three decades: An updated systematic review and meta-regression analysis. Int. J. Epidemiol. 43, 434-442. https://doi.org/10.1093/ije/dyt261 (2014).

10. Hinshaw, S. P. Attention Deficit Hyperactivity Disorder (ADHD): Controversy, developmental mechanisms, and multiple levels of analysis. Annu. Rev. Clin. Psychol. 14, 291-316. https://doi.org/10.1146/annurev-clinpsy-050817-084917 (2018).

11. Bozhilova, N. S., Michelini, G., Kuntsi, J. \& Asherson, P. Mind wandering perspective on attention-deficit/hyperactivity disorder. Neurosci. Biobehav. Rev. 92, 464-476. https://doi.org/10.1016/j.neubiorev.2018.07.010 (2018).

12. Fayyad, J. et al. Cross-national prevalence and correlates of adult attention-deficit hyperactivity disorder. Br. J. Psychiatry 190, 402-409. https://doi.org/10.1192/bjp.bp.106.034389 (2007).

13. Barkley, R. A., Fischer, M., Smallish, L. \& Fletcher, K. The persistence of attention-deficit/hyperactivity disorder into young adulthood as a function of reporting source and definition of disorder. J. Abnorm. Psychol. 111, 279-289 (2002).

14. Pelham, W. E. et al. The long-term financial outcome of children diagnosed with ADHD. J. Consult. Clin. Psychol. 88, 160-171. https://doi.org/10.1037/ccp0000461 (2020).

15. McGee, R., Stanton, W. R. \& Sears, M. R. Allergic disorders and attention deficit disorder in children. J. Abnorm. Child. Psychol. 21, 79-88. https://doi.org/10.1007/bf00910490 (1993).

16. Kaas, T. H. et al. Association between childhood asthma and attention deficit hyperactivity or autism spectrum disorders: A systematic review with meta-analysis. Clin. Exp. Allergy 51, 228-252. https://doi.org/10.1111/cea.13750 (2021).

17. Deckert, S., Kopkow, C. \& Schmitt, J. Nonallergic comorbidities of atopic eczema: an overview of systematic reviews. Allergy 69 , 37-45. https://doi.org/10.1111/all.12246 (2014).

18. Borschuk, A. P., Rodweller, C. \& Salorio, C. F. The influence of comorbid asthma on the severity of symptoms in children with attention-deficit hyperactivity disorder. J. Asthma 55, 66-72. https://doi.org/10.1080/02770903.2017.1306549 (2018).

19. Berzosa-Grande, M. P., González-Fraile, E., Sánchez-López, R., Soria-Oliver, M. \& Rueda-Esteban, S. The relationship between allergic diseases and internalising and externalising behaviours in Spanish children: A cross-sectional study. Allergol. Immunopathol. (Madr) 49, 65-72. https://doi.org/10.15586/aei.v49i3.78 (2021).

20. Hong, S. B. et al. Subthreshold attention-deficit/hyperactivity disorder is associated with functional impairments across domains: A comprehensive analysis in a large-scale community study. Eur. Child Adolesc. Psychiatry 23, 627-636. https://doi.org/10.1007/ s00787-013-0501-z (2014).

21. Schmitt, J., Apfelbacher, C., Heinrich, J., Weidinger, S. \& Romanos, M. Association of atopic eczema and attention-deficit/hyperactivity disorder - meta-analysis of epidemiologic studies. Z. Kinder Jugendpsychiatr. Psychother. 41, 35-42. https://doi.org/10. 1024/1422-4917/a000208 (2013) (quiz 42-34).

22. Schans, J. V., Çiçek, R., de Vries, T. W., Hak, E. \& Hoekstra, P. J. Association of atopic diseases and attention-deficit/hyperactivity disorder: A systematic review and meta-analyses. Neurosci. Biobehav. Rev. 74, 139-148. https://doi.org/10.1016/j.neubiorev.2017. $01.011(2017)$. 
23. He, J. P., Burstein, M., Schmitz, A. \& Merikangas, K. R. The Strengths and Difficulties Questionnaire (SDQ): the factor structure and scale validation in U.S. adolescents. J. Abnorm. Child Psychol. 41, 583-595. https://doi.org/10.1007/s10802-012-9696-6 (2013).

24. Algorta, G. P., Dodd, A. L., Stringaris, A. \& Youngstrom, E. A. Diagnostic efficiency of the SDQ for parents to identify ADHD in the UK: A ROC analysis. Eur. Child Adolesc. Psychiatry 25, 949-957. https://doi.org/10.1007/s00787-015-0815-0 (2016).

25. Vugteveen, J., De Bildt, A., Hartman, C. A. \& Timmerman, M. E. Using the Dutch multi-informant Strengths and Difficulties Questionnaire (SDQ) to predict adolescent psychiatric diagnoses. Eur. Child Adolesc. Psychiatry 27, 1347-1359. https://doi.org/ 10.1007/s00787-018-1127-y (2018).

26. Camfferman, D. et al. Eczema, sleep, and behavior in children. J Clin Sleep Med 6, 581-588 (2010).

27. Mowlem, F. D. et al. Sex differences in predicting ADHD clinical diagnosis and pharmacological treatment. Eur. Child Adolesc. Psychiatry 28, 481-489. https://doi.org/10.1007/s00787-018-1211-3 (2019).

28. Applegate, B. et al. Validity of the age-of-onset criterion for ADHD: a report from the DSM-IV field trials. J. Am. Acad. Child Adolesc. Psychiatry 36, 1211-1221 (1997).

29. Kaas, T. H. et al. Association between childhood asthma and attention deficit hyperactivity or autism spectrum disorders: A systematic review with meta-analysis. Clin. Exp. Allergy https://doi.org/10.1111/cea.13750 (2020).

30. Buske-Kirschbaum, A. et al. Psychoendocrine and psychoneuroimmunological mechanisms in the comorbidity of atopic eczema and attention deficit/hyperactivity disorder. Psychoneuroendocrinology 38, 12-23. https://doi.org/10.1016/j.psyneuen.2012.09.017 (2013).

31. Czarnowicki, T. et al. Early pediatric atopic dermatitis shows only a cutaneous lymphocyte antigen (CLA)(+) TH2/TH1 cell imbalance, whereas adults acquire CLA(+) TH22/TC22 cell subsets. J. Allergy Clin. Immunol. 136, 941-951.e943. https://doi.org/ 10.1016/j.jaci.2015.05.049 (2015).

32. Czarnowicki, T. et al. Evolution of pathologic T-cell subsets in patients with atopic dermatitis from infancy to adulthood. J. Allergy Clin. Immunol. 145, 215-228. https://doi.org/10.1016/j.jaci.2019.09.031 (2020).

33. Looman, K. I. M. et al. Associations of Th2, Th17, Treg cells, and $\operatorname{Ig} \mathrm{A}(+)$ memory B cells with atopic disease in children: The Generation R Study. Allergy 75, 178-187. https://doi.org/10.1111/all.14010 (2020).

34. Puiu, A. A. et al. Impulsive aggression and response inhibition in attention-deficit/hyperactivity disorder and disruptive behavioral disorders: Findings from a systematic review. Neurosci. Biobehav. Rev. 90, 231-246. https://doi.org/10.1016/j.neubiorev.2018.04. 016 (2018).

35. Xing, B., Li, Y. C. \& Gao, W. J. Norepinephrine versus dopamine and their interaction in modulating synaptic function in the prefrontal cortex. Brain Res. 1641, 217-233. https://doi.org/10.1016/j.brainres.2016.01.005 (2016).

36. Kim, J. I. et al. Interaction between DRD2 and lead exposure on the cortical thickness of the frontal lobe in youth with attentiondeficit/hyperactivity disorder. Prog. Neuropsychopharmacol. Biol. Psychiatry 82, 169-176. https://doi.org/10.1016/j.pnpbp.2017. 11.018 (2018).

37. Hiser, J. \& Koenigs, M. The multifaceted role of the ventromedial prefrontal cortex in emotion, decision making, social cognition, and psychopathology. Biol. Psychiatry 83, 638-647. https://doi.org/10.1016/j.biopsych.2017.10.030 (2018).

38. Buske-Kirschbaum, A. et al. Altered hypothalamus-pituitary-adrenal axis function: A relevant factor in the comorbidity of atopic eczema and attention deficit/hyperactivity disorder?. Psychoneuroendocrinology 105, 178-186. https://doi.org/10.1016/j.psyneuen. 2018.12.005 (2019).

39. Park, G., Jung, Y. S., Park, M. K., Yang, C. H. \& Kim, Y. U. Melatonin inhibits attention-deficit/hyperactivity disorder caused by atopic dermatitis-induced psychological stress in an NC/Nga atopic-like mouse model. Sci. Rep. 8, 14981. https://doi.org/10.1038/ s41598-018-33317-x (2018).

40. Xu, Y. C., Wang, J. P., Zhu, W. J. \& Li, P. Childhood atopic dermatitis as a precursor for developing attention deficit/hyperactivity disorder. Int. J. Immunopathol. Pharmacol. 34, 2058738420962902. https://doi.org/10.1177/2058738420962902 (2020).

41. Kuo, H. C., Chang, L. S., Tsai, Z. Y. \& Wang, L. J. Allergic diseases do not impair the cognitive development of children but do damage the mental health of their caregivers. Sci. Rep. 10, 13854. https://doi.org/10.1038/s41598-020-70825-1 (2020).

42. Chamlin, S. L., Frieden, I. J., Williams, M. L. \& Chren, M. M. Effects of atopic dermatitis on young American children and their families. Pediatrics 114, 607-611. https://doi.org/10.1542/peds.2004-0374 (2004).

43. Nicolaides, N. C., Kyratzi, E., Lamprokostopoulou, A., Chrousos, G. P. \& Charmandari, E. Stress, the stress system and the role of glucocorticoids. NeuroImmunoModulation 22, 6-19. https://doi.org/10.1159/000362736 (2015).

44. McCrory, E., De Brito, S. A. \& Viding, E. Research review: the neurobiology and genetics of maltreatment and adversity. J. Child Psychol. Psychiatry 51, 1079-1095. https://doi.org/10.1111/j.1469-7610.2010.02271.x (2010).

45. Lin, T. K., Zhong, L. \& Santiago, J. L. Association between Stress and the HPA Axis in the Atopic Dermatitis. Int J Mol Sci 18, 2131. https://doi.org/10.3390/ijms18102131 (2017).

46. Buske-Kirschbaum, A., Gierens, A., Höllig, H. \& Hellhammer, D. H. Stress-induced immunomodulation is altered in patients with atopic dermatitis. J. Neuroimmunol. 129, 161-167. https://doi.org/10.1016/s0165-5728(02)00168-6 (2002).

47. Quinlan, E. B. et al. Psychosocial stress and brain function in adolescent psychopathology. Am. J. Psychiatry 174, 785-794. https:// doi.org/10.1176/appi.ajp.2017.16040464 (2017).

48. Lau, M. et al. Loss of STAT6 promotes autoimmune disease and atopy on a susceptible genetic background. J. Autoimmun. 39, 388-397. https://doi.org/10.1016/j.jaut.2012.06.003 (2012).

49. Tsai, S. J. Signal transducer and activator of transcription 6 (STAT6) and attention-deficit hyperactivity disorder: A speculative hypothesis. Med. Hypotheses 67, 1342-1344. https://doi.org/10.1016/j.mehy.2006.05.019 (2006).

50. Wamboldt, M. Z. et al. Familial association between allergic disorders and depression in adult Finnish twins. Am. J. Med. Genet. 96, 146-153. https://doi.org/10.1002/(sici)1096-8628(20000403)96:2\%3c146::aid-ajmg4\%3e3.0.co;2-j (2000).

51. Van den Bergh, B. R. H. et al. Prenatal developmental origins of behavior and mental health: The influence of maternal stress in pregnancy. Neurosci. Biobehav. Rev. https://doi.org/10.1016/j.neubiorev.2017.07.003 (2017).

52. Cowell, W. J., Bellinger, D. C., Wright, R. O. \& Wright, R. J. Antenatal active maternal asthma and other atopic disorders is associated with ADHD behaviors among school-aged children. Brain Behav. Immun. 80, 871-878. https://doi.org/10.1016/j.bbi.2019. 05.040 (2019).

53. Roigé-Castellví, J. et al. Prenatal and perinatal factors associated with ADHD risk in schoolchildren: EPINED epidemiological study. Eur. Child Adolesc. Psychiatry https://doi.org/10.1007/s00787-020-01519-2 (2020).

54. Sun, C. K. et al. Therapeutic effects of methylphenidate for attention-deficit/hyperactivity disorder in children with borderline intellectual functioning or intellectual disability: A systematic review and meta-analysis. Sci. Rep. 9, 15908. https://doi.org/10. 1038/s41598-019-52205-6 (2019).

55. Moher, D. et al. Preferred reporting items for systematic review and meta-analysis protocols (PRISMA-P) 2015 statement. Syst. Rev. 4, 1. https://doi.org/10.1186/2046-4053-4-1 (2015).

56. RStudio: Integrated Development for R. (2020).

57. Higgins, J. P. \& Thompson, S. G. Quantifying heterogeneity in a meta-analysis. Stat. Med. 21, 1539-1558. https://doi.org/10.1002/ sim.1186 (2002)

58. Yuksel, H., Sogut, A. \& Yilmaz, O. Attention deficit and hyperactivity symptoms in children with asthma. J. Asthma 45, 545-547. https://doi.org/10.1080/02770900801990016 (2008).

59. Chang, H. Y. et al. Allergic diseases in preschoolers are associated with psychological and behavioural problems. Allergy Asthma Immunol. Res. 5, 315-321. https://doi.org/10.4168/aair.2013.5.5.315 (2013). 
60. Goodwin, R. D. et al. Severity and persistence of asthma and mental health: a birth cohort study. Psychol. Med. 43, 1313-1322. https://doi.org/10.1017/s0033291712001754 (2013).

61. Kim, D. K. et al. Treatment of allergic rhinitis is associated with improved attention performance in children: the Allergic Rhinitis Cohort Study for Kids (ARCO-Kids). PLoS ONE 9, e109145. https://doi.org/10.1371/journal.pone.0109145 (2014).

62. Lee, Y. S. et al. Attention deficit hyperactivity disorder like behavioral problems and parenting stress in pediatric allergic rhinitis. Psychiatry Investig. 11, 266-271. https://doi.org/10.4306/pi.2014.11.3.266 (2014).

63. Yang, M. T. et al. Hyperactivity and impulsivity in children with untreated allergic rhinitis: Corroborated by rating scale and continuous performance test. Pediatr. Neonatol. 55, 168-174. https://doi.org/10.1016/j.pedneo.2013.09.003 (2014).

64. Catal, F. et al. Psychiatric disorders and symptoms severity in preschool children with atopic eczema. Allergol. Immunopathol. 44, 120-124. https://doi.org/10.1016/j.aller.2015.04.006 (2016).

65. Hammer-Helmich, L. et al. Mental health associations with eczema, asthma and hay fever in children: a cross-sectional survey. BMJ Open 6, e012637. https://doi.org/10.1136/bmjopen-2016-012637 (2016).

66. Strom, M. A., Fishbein, A. B., Paller, A. S. \& Silverberg, J. I. Association between atopic dermatitis and attention deficit hyperactivity disorder in U.S. children and adults. Br. J. Dermatol. 175, 920-929. https://doi.org/10.1111/bjd.14697 (2016).

67. Yang, M. T. et al. Attention-deficit/hyperactivity disorder-related symptoms improved with allergic rhinitis treatment in children. Am. J. Rhinol. Allergy 30, 209-214. https://doi.org/10.2500/ajra.2016.30.4301 (2016).

68. Feng, B. et al. Association of pediatric allergic rhinitis with the ratings of attention-deficit/hyperactivity disorder. Am. J. Rhinol. Allergy 31, 161-167. https://doi.org/10.2500/ajra.2017.31.4439 (2017).

69. Abd El-Hamid, Z. B. et al. Impact of allergy on children with attention deficit hyperactivity disorder. Eur. Ann. Allergy Clin. Immunol. 50, 262-267. https://doi.org/10.23822/EurAnnACI.1764-1489.72 (2018).

70. Kuniyoshi, Y. et al. Severity of eczema and mental health problems in Japanese schoolchildren: The ToMMo Child Health Study. Allergol. Int. 67, 481-486. https://doi.org/10.1016/j.alit.2018.02.009 (2018).

71. Kruse, L. L., Cices, A., Fishbein, A. B. \& Paller, A. S. Neurocognitive function in moderate-severe pediatric atopic dermatitis: A case-control study. Pediatr. Dermatol. 36, 110-114. https://doi.org/10.1111/pde.13710 (2019).

72. Tajdini, M. et al. Associations of behavioral disorders with asthma in Iranian children. Iran J. Allergy Asthma Immunol. 18, 340-345. https://doi.org/10.18502/ijaai.v18i3.1127 (2019).

73. Zhou, H., Chen, Z., Zhao, W., Liu, Y. \& Cui, Y. Evaluation of neuropsychiatric comorbidities and their clinical characteristics in Chinese children with asthma using the MINI kid tool. BMC Pediatr 19, 454. https://doi.org/10.1186/s12887-019-1834-7 (2019).

74. Feng, L. J., Chen, A. W., Luo, X. Y. \& Wang, H. Increased attention deficit/hyperactivity and oppositional defiance symptoms of 6-12 years old Chinese children with atopic dermatitis. Medicine (Baltimore) 99, e20801. https://doi.org/10.1097/MD.0000000000 $020801(2020)$

75. Fuhrmann, S., Tesch, F., Romanos, M., Abraham, S. \& Schmitt, J. ADHD in school-age children is related to infant exposure to systemic H1-antihistamines. Allergy 75, 2956-2957. https://doi.org/10.1111/all.14411 (2020).

76. Guo, M. M., Wang, L. J., Hsu, T. Y., Yang, K. D. \& Kuo, H. C. Peanut sensitivity and allergic rhinitis in young children are associated with attention-deficit hyperactivity disorder symptoms in adolescence. Neuropsychiatr. Dis. Treat. 16, 1349-1357. https://doi.org/ 10.2147/NDT.S232299 (2020).

77. Huang, A. H. et al. Real-world comorbidities of atopic dermatitis in the pediatric ambulatory population in the United States. J. Am. Acad. Dermatol. https://doi.org/10.1016/j.jaad.2021.03.016 (2021).

78. Jackson-Cowan, L., Cole, E. F., Silverberg, J. I. \& Lawley, L. P. Childhood atopic dermatitis is associated with cognitive dysfunction: A National Health Interview Survey study from 2008 to 2018. Ann. Allergy Asthma Immunol. 126, 661-665. https://doi.org/10. 1016/j.anai.2020.11.008 (2021).

79. Minatoya, M., Suyama, S. \& Kishi, R. Relationship between atopic dermatitis and children's mental and behavioral health: The Hokkaido Study. Nihon Koshu Eisei Zasshi 67, 745-751. https://doi.org/10.11236/jph.67.10_745 (2020).

80. Montalbano, L. et al. Relationship between quality of life and behavioural disorders in children with persistent asthma: A Multiple Indicators Multiple Causes (MIMIC) model. Sci. Rep. 10, 6957. https://doi.org/10.1038/s41598-020-62264-9 (2020).

81. Edvinsson Sollander, S. et al. Asthma and allergies correlate with mental health problems in preschool children. Acta Paediatr. 110, 1601-1609. https://doi.org/10.1111/apa.15709 (2021).

82. Wan, J., Takeshita, J., Shin, D. B. \& Gelfand, J. M. Mental health impairment among children with atopic dermatitis: A United States population-based cross-sectional study of the 2013-2017 National Health Interview Survey. J. Am. Acad. Dermatol. 82, 1368-1375. https://doi.org/10.1016/j.jaad.2019.10.019 (2020).

83. Chai, P. H., Chang, S. \& Cawthorpe, D. The temporal hyper-morbidity of asthma and attention deficit disorder: Implications for interpretation based on comparison of prospective and cross-sectional population samples. Psychiatry Investig. 18, 166-171. https:// doi.org/10.30773/pi.2020.0349 (2021).

84. Galéra, C. et al. Medical conditions and Attention-Deficit/Hyperactivity Disorder symptoms from early childhood to adolescence. Mol. Psychiatry https://doi.org/10.1038/s41380-021-01357-x (2021).

85. Hou, A. \& Silverberg, J. I. Predictors and age-dependent pattern of psychologic problems in childhood atopic dermatitis. Pediatr. Dermatol. 38, 606-612. https://doi.org/10.1111/pde.14588 (2021).

86. Keller, W. et al. Atopic diseases in children and adolescents are associated with behavioural difficulties. BMC Pediatr. https://doi. org/10.1186/s12887-021-02663-7 (2021).

87. Özyurt, G. et al. Adolescents with asthma reported more peer victimization, more anger repression, and less anger expression. J. Asthma 58, 1307-1313. https://doi.org/10.1080/02770903.2020.1782428 (2021).

88. Rajhans, P., Sagar, R., Patra, B. N., Bhargava, R. \& Kabra, S. K. Psychiatric morbidity and behavioral problems in children and adolescents with bronchial asthma. Indian J. Pediatr. 88, 968-973. https://doi.org/10.1007/s12098-021-03661-4 (2021).

89. Vittrup, I. et al. Association between hospital-diagnosed atopic dermatitis and psychiatric disorders and medication use in childhood. Br. J. Dermatol. 185, 91-100. https://doi.org/10.1111/bjd.19817 (2021).

90. Yüksel, A. E. et al. Adhd and its associations with pregnancy, birth, developmental and medical-related characteristics. Curr. Psychol. https://doi.org/10.1007/s12144-021-01817-1 (2021).

\section{Acknowledgements}

We would like to acknowledge Yi-No Kang for his help and useful suggestions related to statistical measurements. This manuscript was edited by Wallace Academic Editing.

\section{Author contributions}

Conceived and designed the experiments: Yu-chieh Chuang, Yu-Jui Huang, Yang-Ching Chen/Analyzed the data: Yu-Chieh Chuang, Liang-Jen Wang, Yang-Ching Chen/Wrote the paper: Yu-Chieh Chuang, Yu-Jui Huang, Wei-Lieh Huang/Data acquisition, analysis and interpretation; manuscript drafting, editing and submission: Wei-Lieh Huang, Ching-Yun Wang, Ho-Chang Kuo/Abstracts review, evaluation of inclusion criteria and bias analysis: Wei-Lieh Huang, Ching-Yun Wang, Ho-Chang Kuo/All authors reviewed the manuscript. 


\section{Competing interests}

The authors declare no competing interests.

\section{Additional information}

Supplementary Information The online version contains supplementary material available at https://doi.org/ 10.1038/s41598-022-07232-1.

Correspondence and requests for materials should be addressed to Y.-J.H.

Reprints and permissions information is available at www.nature.com/reprints.

Publisher's note Springer Nature remains neutral with regard to jurisdictional claims in published maps and institutional affiliations.

(c) (1) Open Access This article is licensed under a Creative Commons Attribution 4.0 International License, which permits use, sharing, adaptation, distribution and reproduction in any medium or format, as long as you give appropriate credit to the original author(s) and the source, provide a link to the Creative Commons licence, and indicate if changes were made. The images or other third party material in this article are included in the article's Creative Commons licence, unless indicated otherwise in a credit line to the material. If material is not included in the article's Creative Commons licence and your intended use is not permitted by statutory regulation or exceeds the permitted use, you will need to obtain permission directly from the copyright holder. To view a copy of this licence, visit http://creativecommons.org/licenses/by/4.0/.

(c) The Author(s) 2022 